\title{
Modelling of subgrid-scale phenomena in supercritical transitional mixing layers: an a priori study
}

\author{
LAURENT C. SELLE ${ }^{1}$, NORA A. OKONG'O', \\ JOSETTE BELLAN ${ }^{1,2}$ AND KENNETH G. HARSTAD \\ ${ }^{1}$ California Institute of Technology, Pasadena, CA 91125, USA \\ ${ }^{2}$ Jet Propulsion Laboratory, California Institute of Technology, \\ Pasadena, CA 91109, USA
}

(Received 27 October 2006 and in revised form 25 June 2007)

A database of transitional direct numerical simulation (DNS) realizations of a supercritical mixing layer is analysed for understanding small-scale behaviour and examining subgrid-scale (SGS) models duplicating that behaviour. Initially, the mixing layer contains a single chemical species in each of the two streams, and a perturbation promotes roll-up and a double pairing of the four spanwise vortices initially present. The database encompasses three combinations of chemical species, several perturbation wavelengths and amplitudes, and several initial Reynolds numbers specifically chosen for the sole purpose of achieving transition. The DNS equations are the Navier-Stokes, total energy and species equations coupled to a real-gas equation of state; the fluxes of species and heat include the Soret and Dufour effects. The large-eddy simulation (LES) equations are derived from the DNS ones through filtering. Compared to the DNS equations, two types of additional terms are identified in the LES equations: SGS fluxes and other terms for which either assumptions or models are necessary. The magnitude of all terms in the LES conservation equations is analysed on the DNS database, with special attention to terms that could possibly be neglected. It is shown that in contrast to atmospheric-pressure gaseous flows, there are two new terms that must be modelled: one in each of the momentum and the energy equations. These new terms can be thought to result from the filtering of the nonlinear equation of state, and are associated with regions of high densitygradient magnitude both found in DNS and observed experimentally in fully turbulent high-pressure flows. A model is derived for the momentum-equation additional term that performs well at small filter size but deteriorates as the filter size increases, highlighting the necessity of ensuring appropriate grid resolution in LES. Modelling approaches for the energy-equation additional term are proposed, all of which may be too computationally intensive in LES. Several SGS flux models are tested on an a priori basis. The Smagorinsky (SM) model has a poor correlation with the data, while the gradient (GR) and scale-similarity (SS) models have high correlations. Calibrated model coefficients for the GR and SS models yield good agreement with the SGS fluxes, although statistically, the coefficients are not valid over all realizations. The GR model is also tested for the variances entering the calculation of the new terms in the momentum and energy equations; high correlations are obtained, although the calibrated coefficients are not statistically significant over the entire database at fixed filter size. As a manifestation of the small-scale supercritical mixing peculiarities, both scalar-dissipation visualizations and the scalar-dissipation probability density functions (PDF) are examined. The PDF is shown to exhibit minor peaks, with 
particular significance for those at larger scalar dissipation values than the mean, thus significantly departing from the Gaussian behaviour.

\section{Introduction}

Supercritical fluids are of great interest in extraction processes as well as in propulsion devices such as advanced gas-turbine and diesel engines, and liquid rockets. The performance of these devices depends on the efficiency of fluid disintegration and turbulent mixing. As discussed in detail by Harstad \& Bellan (2001), consistency with single-species thermodynamics requires that a fluid be defined to be in a supercritical state when it is at a thermodynamic pressure, $p$, or temperature, $T$, exceeding its critical (subscript $c$ ) value $p_{c}$ or $T_{c}$ (Prausnitz, Lichtenhaler \& de Azevedo 1986); therefore, in the supercritical regime there is no longer the possibility of a two-phase (i.e. gas/liquid) region (Hirshfelder, Curtis \& Bird 1964). For mixtures, both $p_{c}$ and $T_{c}$ depend on the composition. The present interest is in fluid mixtures at high pressures that are supercritical for the pure species. Past the critical point of the fluid (where material surfaces no longer exist), the disintegration of fluid jets displays an aspect that Chehroudi, Talley \& Coy (1999) call 'fingers' or 'comb-like structures' at transcritical conditions, which have an increasingly gaseous appearance with increasing $p$. Similar experimental evidence was produced by Mayer et al. (1996) and Mayer et al. (1998) for $\mathrm{O}_{2}$ disintegration. For supercritical free $\mathrm{N}_{2}$ jets, the experiments of Oschwald \& Schik (1999) also highlighted sharp density profiles, indicating the occurrence of high density gradients.

Results from direct numerical simulations (DNS) of temporal mixing layers under supercritical conditions showed that regions of high density-gradient magnitude (HDGM), akin to those in the experiments, exist in both pre-transitional (Miller, Harstad \& Bellan 2001) and transitional (Okong'o \& Bellan 2002a; Okong'o, Harstad \& Bellan 2002) temporal mixing layers, arising both from the initial density stratification and from mixing (Okong'o \& Bellan 2004a). These DNS were conducted using a real-gas equation of state for non-ideal mixtures in conjunction with realistic transport properties and thermal diffusion (Soret and Dufour) effects. For modelling fully turbulent supercritical flows at high pressures, large-eddy simulation (LES), wherein only the large scales are simulated and the subgrid scales (SGS) are modelled, at present seems more computationally achievable for practical systems than DNS, which requires all turbulence scales to be resolved. The LES equations are derived by applying a spatial filter to the DNS equations, leading to various unclosed terms, including the SGS fluxes, which arise from filtering the convective terms. Given the distinctive supercritical flow characteristics, it is of interest to inquire whether LES models developed for compressible perfect-gas and incompressible flows can be extended to real-gas non-ideal mixtures.

So far, LES of supercritical flows have been conducted with equations similar to those for atmospheric-pressure gaseous flows; mathematically, these are the DNS equations with the addition of SGS fluxes (Oefelein \& Yang 1998; Zong et al. 2004; Oefelein 2005), for which primarily a Smagorinsky type of SGS flux model (Smagorinksy 1993) was used. The strategy of the present study is to analyse, on an a priori basis, DNS databases for transitional supercritical temporal mixing layers (including those in Okong'o \& Bellan 2002a; Okong'o et al. 2002) to inquire whether the atmospheric-pressure equations previously used are still correct in the realm of thermodynamically supercritical flows. We show here that under supercritical 
conditions requiring the utilization of a real-gas equation of state (EOS) (Harstad \& Bellan 2000; Okong'o \& Bellan 2002a), the strictly low-pressure LES equations are no longer valid as new significant terms arise from the filtering of the conservation equations; these terms are directly associated with the HDGM regions observed both in simulations and experimentally, meaning that the validity of the novel terms extends to higher Reynolds number values than those in the transitional databases. Noteworthy, because real-gas EOS are nonlinear, even small departures from perfect-gas or ideal mixture (i.e. slight real-gas or non-ideal mixture) behaviour, imparts departures from the atmospheric-pressure LES equations; this is the nature of nonlinearity. We also show that, similar to atmospheric gaseous flow (Okong'o \& Bellan 2004b), the Smagorinsky model is a poor approximation of the SGS fluxes, for which other models are found to be more successful. In $\S 2$, the LES governing equations are presented, and the unclosed terms that must be modelled are identified. The databases are summarized in $\S 3$, followed by the $a$ priori analysis of the database in $\S 4$. The analysis includes assessment of the simplifying assumptions for the unclosed terms that are not SGS fluxes and explicit modelling of the SGS fluxes. The scalar dissipation, originating from small-scale mixing and contributing overwhelmingly to the total dissipation (Okong'o \& Bellan 2002a), is analysed as an example of phenomena that must be captured in LES by the SGS models, further justifying our proposed modelling approach. Finally, $\S 5$ contains the conclusions and areas of future endeavour.

\section{Governing equations for large-eddy simulations}

The LES equations are derived from the DNS set by spatial filtering. The filtering operation is defined as

$$
\bar{\psi}(\boldsymbol{x})=\int_{V} \psi(\boldsymbol{y}) G(\boldsymbol{x}-\boldsymbol{y}) \mathrm{d} \boldsymbol{y}
$$

where $G$ is the filter function and $V$ is the filtering volume; $G$ has the property that for a spatially invariant function, the filtered function is identical to the unfiltered one. For compressible flows, Favre filtering is used, defined as $\tilde{\psi}=\overline{\rho \psi} / \bar{\rho}$ where $\rho$ is the density. The variance of two quantities $\varphi$ and $\theta$ is defined as $\vartheta(\bar{\varphi}, \bar{\theta})=\overline{\varphi \theta}-\bar{\varphi} \bar{\theta}$ or $\vartheta(\widetilde{\varphi}, \widetilde{\theta})=\widetilde{\varphi \theta}-\widetilde{\varphi} \widetilde{\theta}$, depending on the filtering. The governing equations are written for the conservative variables $\phi=\left\{\rho, \rho u_{i}, \rho e_{t}, \rho Y_{\alpha}\right\}$ rather than the primitive variables $\psi(\phi)=\left\{u_{i}, p, X_{\alpha}, T\right\}$ or $\psi^{\prime}(\phi)=\left\{u_{i}, v, X_{\alpha}, T\right\}$, where $u_{i}$ is the velocity component in the $x_{i}$-direction, $e_{t}$ is the total energy, $v$ is the molar volume, and $Y_{\alpha}$ and $X_{\alpha}$ are the mass fraction and mole fraction of species $\alpha$.

\subsection{DNS equations}

The conservation equations for a mixture of $N$ species are

$$
\begin{gathered}
\frac{\partial \rho}{\partial t}+\frac{\partial \rho u_{j}}{\partial x_{j}}=0 \\
\frac{\partial\left(\rho u_{i}\right)}{\partial t}+\frac{\partial\left(\rho u_{i} u_{j}\right)}{\partial x_{j}}=-\frac{\partial p}{\partial x_{i}}+\frac{\partial \sigma_{i j}}{\partial x_{j}}, \\
\frac{\partial\left(\rho e_{t}\right)}{\partial t}+\frac{\partial\left(\rho e_{t} u_{j}\right)}{\partial x_{j}}=-\frac{\partial\left(p u_{j}\right)}{\partial x_{j}}-\frac{\partial q_{I K j}}{\partial x_{j}}+\frac{\partial\left(\sigma_{i j} u_{i}\right)}{\partial x_{j}},
\end{gathered}
$$




$$
\frac{\partial\left(\rho Y_{\alpha}\right)}{\partial t}+\frac{\partial\left(\rho Y_{\alpha} u_{j}\right)}{\partial x_{j}}=-\frac{\partial j_{\alpha j}}{\partial x_{j}}
$$

where $t$ is the time, $\boldsymbol{\sigma}$ is the viscous stress tensor, $\boldsymbol{q}_{I K}$ is the Irwing-Kirkwood (subscript $I K$ ) heat flux, $e=e_{t}-e_{K}$ is the internal energy, $e_{K}=u_{i} u_{i} / 2$ is the kinetic energy, and $\boldsymbol{j}_{\alpha}$ is the species-mass flux of species $\alpha$. Also,

$$
\sum_{\alpha=1}^{N} Y_{\alpha}=1, \quad \sum_{\alpha=1}^{N} j_{\alpha j}=0 .
$$

Here, the Einstein summation is used for roman indices $(i, j, k)$, but not for Greek indices $(\alpha, \beta)$. The thermodynamic variables are functions of the flow field $\phi$ :

$$
e=e(\phi), \quad p=p(\phi), \quad T=T(\phi), \quad h=h(\phi),
$$

where $p, T$ and the enthalpy $h=e+p / \rho$ are computed from the EOS; likewise, the fluxes are functions of $\phi$ :

$$
\sigma_{i j}=\sigma_{i j}(\phi), \quad j_{\alpha j}=j_{\alpha j}(\phi), \quad q_{I K j}=q_{I K j}(\phi) .
$$

For a Newtonian fluid,

$$
\sigma_{i j}=\mu\left(\frac{\partial u_{i}}{\partial x_{j}}+\frac{\partial u_{j}}{\partial x_{i}}-\frac{2}{3} \frac{\partial u_{k}}{\partial x_{k}} \delta_{i j}\right), S_{i j}=\frac{1}{2}\left(\frac{\partial u_{i}}{\partial x_{j}}+\frac{\partial u_{j}}{\partial x_{i}}\right)
$$

where $\mu$ is the viscosity and $S_{i j}$ is the rate-of-strain tensor.

The species-mass and heat fluxes originate in the fluctuation-dissipation theory (see Keizer 1987) which is consistent with non-equilibrium thermodynamics, converges to kinetic theory in the low-pressure limit and relates fluxes and forces from first principles. For a binary-species system (light species 1 , heavy species 2 ), the speciesmass and heat fluxes, including Soret and Dufour effects (Harstad \& Bellan 2000), are

$$
\begin{aligned}
\boldsymbol{j}_{2}(\psi) & =B_{Y}(\psi) \nabla Y_{2}+B_{T}(\psi) \nabla T+B_{P}(\psi) \nabla p \\
\boldsymbol{q}_{I K}(\psi) & =C_{Y}(\psi) \nabla Y_{2}+C_{T}(\psi) \nabla T+C_{P}(\psi) \nabla p
\end{aligned}
$$

where

$$
\begin{aligned}
& B_{Y} \equiv-\rho D \alpha_{D}, \quad C_{Y} \equiv-\rho D \alpha_{D} \alpha_{I K} R_{u} T \frac{m}{m_{1} m_{2}}, \\
& B_{T} \equiv-\alpha_{B K} Y_{1} Y_{2} \frac{\rho D}{T}, \quad C_{T} \equiv-\lambda-\rho D \alpha_{I K} \alpha_{B K} R_{u} \frac{m}{m_{1} m_{2}} Y_{1} Y_{2}, \\
& B_{P} \equiv-\rho D \frac{Y_{1} Y_{2}}{R_{u} T} \frac{m_{2} m_{1}}{m} \Lambda, C_{P} \equiv-\rho D \alpha_{I K} \Lambda Y_{1} Y_{2}
\end{aligned}
$$

with

$$
\begin{gathered}
\alpha_{B K}=\alpha_{I K}-\alpha_{h}, \quad \alpha_{h}=\frac{1}{R_{u} T} \frac{m_{2} m_{1}}{m} \Theta \\
\Lambda \equiv\left(\frac{1}{m_{2}} \frac{\partial v}{\partial X_{2}}-\frac{1}{m_{1}} \frac{\partial v}{\partial X_{1}}\right), \quad \Theta \equiv\left(\frac{1}{m_{2}} \frac{\partial(m h)}{\partial X_{2}}-\frac{1}{m_{1}} \frac{\partial(m h)}{\partial X_{1}}\right) \\
\alpha_{D} \equiv 1+X_{\alpha} \frac{\partial \ln \gamma_{\alpha}}{\partial X_{\alpha}}, \quad \alpha=1 \text { or } 2
\end{gathered}
$$


where $\lambda$ is the thermal conductivity with $\lim _{p \rightarrow 0} \lambda=\lambda_{K T}$ as discussed in Harstad \& Bellan (2000) where the subscript $K T$ denotes the kinetic theory, $R_{u}$ is the universal gas constant, $m$ is the mixture molar mass, and $v=m / \rho$. For species $\alpha, m_{\alpha}$ is the species- $\alpha$ molar mass, $X_{\alpha}=m Y_{\alpha} / m_{\alpha}$, and $\gamma_{\alpha}$ is the fugacity. Furthermore, $\alpha_{I K}$ is the IK form of the thermal diffusion factor, $\alpha_{B K}$ is the Bearman-Kirkwood (BK) form of the thermal diffusion factor, $D$ is the binary diffusion coefficient and $\alpha_{D}$ is the mass diffusion factor.

\subsection{LES equations}

After filtering, and assuming that filtering and differentiation commute (the top-hat filter is used here for which the operations commute except near boundaries), the governing equations become

$$
\begin{gathered}
\frac{\partial \bar{\rho}}{\partial t}+\frac{\partial \bar{\rho} \tilde{u}_{j}}{\partial x_{j}}=0, \\
\frac{\partial \bar{\rho} \tilde{u}_{i}}{\partial t}+\frac{\partial \overline{\rho u_{i} u_{j}}}{\partial x_{j}}=-\frac{\partial \bar{p}}{\partial x_{i}}+\frac{\partial \bar{\sigma}_{i j}}{\partial x_{j}}, \\
\frac{\partial \bar{\rho} \tilde{e_{t}}}{\partial t}+\frac{\partial \overline{\rho e_{t} u_{j}}}{\partial x_{j}}=-\frac{\partial \overline{p u_{j}}}{\partial x_{j}}-\frac{\partial \bar{q}_{I K j}}{\partial x_{j}}+\frac{\partial \overline{\sigma_{i j} u_{i}}}{\partial x_{j}}, \\
\frac{\partial \bar{\rho} \tilde{Y}_{\alpha}}{\partial t}+\frac{\partial \frac{\partial Y_{\alpha} u_{j}}{\partial x_{j}}}{=}=-\frac{\partial \bar{j}_{\alpha j}}{\partial x_{j}}, \\
\tilde{e_{t}}=\tilde{e}+\widetilde{u_{i} u_{i}} / 2, \quad \sum_{\alpha=1}^{N} \tilde{Y}_{\alpha j}=1, \quad \sum_{\alpha=1}^{N} \bar{j}_{\alpha j}=0 .
\end{gathered}
$$

With $\phi$ denoting the DNS flow field, the filtered flow field is now denoted as $\bar{\phi}$, and one can define functions of $\bar{\phi}$ :

$$
e(\bar{\phi}), \quad p(\bar{\phi}), \quad T(\bar{\phi}), \quad h(\bar{\phi}), \quad \sigma_{i j}(\bar{\phi}), \quad j_{\alpha j}(\bar{\phi}), \quad q_{I K j}(\bar{\phi}),
$$

which have the same functional form as in the DNS and that in general differ from their filtered counterparts

$$
\begin{array}{cl}
\tilde{e}=\overline{\rho e(\phi)} / \bar{\rho}, & \bar{p}=\overline{p(\phi)}, \quad \bar{T}=\overline{T(\phi)}, \quad \tilde{T}=\overline{\rho T(\phi)} / \bar{\rho}, \quad \tilde{h}=\overline{\rho h(\phi)} / \bar{\rho}, \\
\bar{\sigma}_{i j}=\overline{\sigma_{i j}(\phi)}, \bar{j}_{\alpha j}=\overline{j_{\alpha j}(\phi)}, \quad \bar{q}_{I K j}=\overline{q_{I K j}(\phi)} .
\end{array}
$$

Defining the SGS fluxes,

$$
\tau_{i j}=\vartheta\left(\widetilde{u}_{i}, \widetilde{u}_{j}\right), \quad \zeta_{j}=\vartheta\left(\tilde{h}, \tilde{u}_{j}\right), \quad \eta_{\alpha j}=\vartheta\left(\widetilde{Y}_{\alpha}, \widetilde{u}_{j}\right) \quad \text { with } \quad \sum_{\alpha=1}^{N} \eta_{\alpha j}=0
$$

the filtered governing equations are

$$
\begin{gathered}
\frac{\partial \bar{\rho}}{\partial t}+\frac{\partial \bar{\rho} \tilde{u}_{j}}{\partial x_{j}}=0 \\
\frac{\partial \bar{\rho} \tilde{u}_{i}}{\partial t}+\frac{\partial \bar{\rho} \tilde{u}_{i} \tilde{u}_{j}}{\partial x_{j}}=-\frac{\partial p(\bar{\phi})}{\partial x_{i}}+\frac{\partial \sigma_{i j}(\bar{\phi})}{\partial x_{j}}-\frac{\partial}{\partial x_{j}}\left(\bar{\rho} \tau_{i j}\right) \\
-\frac{\partial}{\partial x_{i}}[\bar{p}-p(\bar{\phi})]+\frac{\partial}{\partial x_{j}}\left[\bar{\sigma}_{i j}-\sigma_{i j}(\bar{\phi})\right],
\end{gathered}
$$




$\begin{array}{lccc}\text { Species } & m(\mathrm{~g} / \mathrm{mol}) & T_{c}(\mathrm{~K}) & p_{c}(\mathrm{MPa}) \\ \mathrm{H}_{2} & 2.016 & 33.0 & 1.284 \\ \mathrm{He} & 4.003 & 5.19 & 0.227 \\ \mathrm{~N}_{2} & 28.013 & 126.3 & 3.399 \\ \mathrm{O}_{2} & 31.999 & 154.6 & 5.043 \\ \mathrm{C}_{7} \mathrm{H}_{16} & 100.205 & 540.2 & 2.74\end{array}$

TABle 1. Pure species properties.

$$
\begin{gathered}
\frac{\partial \bar{\rho} \tilde{e}_{t}}{\partial t}+\frac{\partial \tilde{\rho} \tilde{e}_{t} \tilde{u}_{j}}{\partial x_{j}}=-\frac{\partial p(\bar{\phi}) \tilde{u}_{j}}{\partial x_{j}}-\frac{\partial q_{I K j}(\bar{\phi})}{\partial x_{j}}+\frac{\partial \sigma_{i j}(\bar{\phi}) \tilde{u}_{i}}{\partial x_{j}}-\frac{\partial}{\partial x_{j}}\left(\bar{\rho} \zeta_{j}\right)-\frac{\partial}{\partial x_{j}}\left(\bar{\rho} \kappa_{j}\right) \\
-\frac{\partial}{\partial x_{j}}\left\{[\bar{p}-p(\bar{\phi})] \tilde{u}_{j}\right\}-\frac{\partial}{\partial x_{j}}\left[\bar{q}_{I K j}-q_{I K j}(\bar{\phi})\right]+\frac{\partial}{\partial x_{j}}\left[\overline{\sigma_{i j} u_{i}}-\sigma_{i j}(\bar{\phi}) \tilde{u}_{i}\right] \\
\frac{\partial \bar{\rho} \tilde{Y}_{\alpha}}{\partial t}+\frac{\partial \bar{\rho} \tilde{Y}_{\alpha} \tilde{u}_{j}}{\partial x_{j}}=-\frac{\partial j_{\alpha j}(\bar{\phi})}{\partial x_{j}}-\frac{\partial}{\partial x_{j}}\left(\bar{\rho} \eta_{\alpha j}\right)-\frac{\partial}{\partial x_{j}}\left[\bar{j}_{\alpha j}-j_{\alpha j}(\bar{\phi})\right]
\end{gathered}
$$

where $\kappa_{j}=\vartheta\left(\widetilde{e}_{K}, \widetilde{u}_{j}\right)$. These equations contain several unclosed terms that cannot be directly computed from the filtered flow field. To compute these terms, two closure approaches are pursued: explicit models for the SGS fluxes, and simplifying assumptions for the remaining terms. In particular, it is noted that even if, for example, $\bar{p} \simeq p(\bar{\phi})$, this may not mean that the corresponding term in equation (2.28) is small because that term is $\nabla[\bar{p}-p(\bar{\phi})]$ which may vary differently from $[\bar{p}-p(\bar{\phi})]$. The assumptions and models are assessed in $\S 4$ on a DNS database, described below, of a binary non-reacting temporal mixing layer.

\section{Description of DNS database}

The database consists of supercritical temporal mixing layer simulations of binary $(N=2)$ mixtures, namely, heptane/nitrogen $(\mathrm{HN})$, oxygen/hydrogen $(\mathrm{OH})$ and oxygen/helium $(\mathrm{OHe})$. The pure species properties are listed in table 1. For each layer, species 1 and 2 initially reside in the upper and lower stream, respectively.

A detailed description of the DNS methodology has been given by Miller et al. (2001) and Okong'o \& Bellan (2002a) for HN layers and by Okong'o, Harstad \& Bellan (2002) and Okong'o \& Bellan (2003) for the $\mathrm{OH}$ layers. The conservation equations were numerically solved using a fourth-order explicit Runge-Kutta time integration and a sixth-order compact scheme with eighth-order filter for spatial derivatives (Kennedy \& Carpenter 1994); the filtering (applied at interior points only) is required to maintain numerical stability for long-time integrations but since it acts only on the shortest waves that can be resolved on the grid, it does not act as a turbulence model allowing under-resolved computations. The computations were parallelized using three-dimensional domain decomposition and message passing, and an efficient parallel tridiagonal solver (Muller \& Scheerer 1991). The configuration, initial and boundary conditions, EOS, and transport property relations are summarized below.

\subsection{Configuration, initial and boundary conditions}

The temporally developing mixing layer configuration is depicted in figure 1 for $\mathrm{HN}$, as an example, showing the definition of the streamwise $\left(x_{1}\right)$, cross-stream $\left(x_{2}\right)$ and 


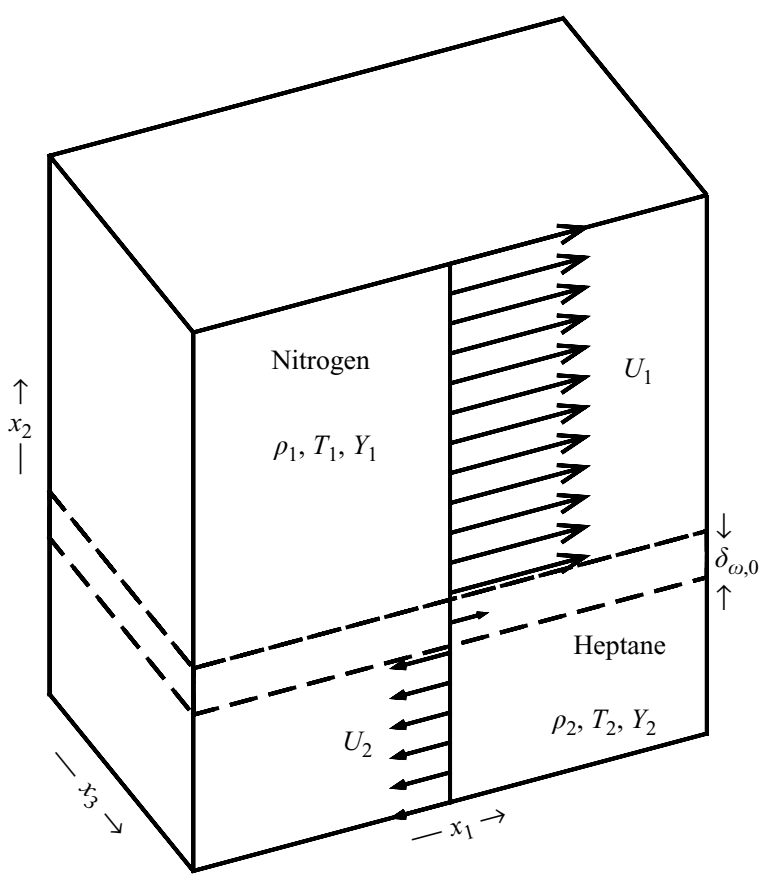

FIgURE 1. $\mathrm{C}_{7} \mathrm{H}_{16} / \mathrm{N}_{2}$ mixing-layer configuration.

spanwise $\left(x_{3}\right)$ coordinates. The layer is not symmetric in extent in the $x_{2}$-direction, to accommodate the larger layer growth on the lighter fluid side. The free-stream density $\left(\rho_{1}\right.$ or $\left.\rho_{2}\right)$ is calculated for each pure species at its free-stream temperature $\left(T_{1}\right.$ or $\left.T_{2}\right)$ and at the initial uniform pressure $\left(p_{0}\right)$. The vorticity thickness is defined as $\delta_{\omega}(t)=\Delta U_{0} /\left(\partial\left\langle u_{1}\right\rangle / \partial x_{2}\right)_{\max }$ where $\left\langle u_{1}\right\rangle$ is the $\left(x_{1}, x_{3}\right)$ planar average of the streamwise velocity, and $\Delta U_{0}=U_{1}-U_{2}$ is the velocity difference across the layer. $U_{1}$ and $U_{2}$ were chosen with the intent of keeping the ultimate vortex stationary in the computational domain (Miller et al. 2001; Papamoschou \& Roshko 1988); the specification of the convective Mach number, $M_{c, 0}$, determines $\Delta U_{0}$. Given the initial streamwise velocity profile $u_{1}$ based on $U_{1}$ and $U_{2},\left(\partial\left\langle u_{1}\right\rangle / \partial x_{2}\right)_{\max }$ and hence $\delta_{\omega, 0} \equiv \delta_{\omega}(0)$ are calculated. The initial momentum ratio $\left|\rho_{2} U_{2}\right| /\left|\rho_{1} U_{1}\right| \sim 5$ for all $\mathrm{HN}$ and $\mathrm{OH}$ simulations and $\sim 3.5$ for the $\mathrm{OHe}$ simulation for which the choice of initial conditions that matched in temperature those of an $\mathrm{OH}$ simulation prevented reaching the same value of $\left|\rho_{2} U_{2}\right| /\left|\rho_{1} U_{1}\right|$. The specified value of the initial flow Reynolds number, $R e_{0}=(1 / 2)\left(\rho_{1}+\rho_{2}\right) \Delta U_{0} \delta_{\omega, 0} / \mu_{R}$, chosen so as to enable the resolution of all relevant length scales, is then used to calculate $\mu_{R}$, which scales $\mu$. The grid spacing is an approximately linear function of $R e_{0}$.

The simulations are started with error-function profiles for the mean streamwise velocity, mass fraction and temperature, upon which are imposed spanwise and streamwise vorticity perturbations (Moser \& Rogers 1991, 1993) of strengths $F_{2 D}$ and $F_{3 D}$ respectively, whose streamwise $\left(\lambda_{1}\right)$ and spanwise $\left(\lambda_{3}\right)$ wavelengths are $\lambda_{1}=C \delta_{\omega, 0}$ and $\lambda_{3}=0.6 \lambda_{1}$, where $C=7.29$ is the most unstable wavelength for incompressible flow. For the simulations reported here, listed in table 2, other values of $C$ obtained from stability analyses (Okong'o \& Bellan 2003) were also used: $C=4.57$ for the shortest (estimated) unstable wavelength for the HN layer, or $C$ corresponding to the most unstable wavelength for $\mathrm{O}_{2}$ layers. The grid is chosen for all simulations so as 


\begin{tabular}{lcccccccc}
\hline Run & $\mathrm{HN} 400$ & $\mathrm{HN} 500$ & $\mathrm{HN} 600$ & $\mathrm{HN} 800$ & $\mathrm{OH} 750$ & $\mathrm{OH} 550$ & $\mathrm{OH} 500$ & OHe600 \\
Species 2 & $\mathrm{C}_{7} \mathrm{H}_{16}$ & $\mathrm{C}_{7} \mathrm{H}_{16}$ & $\mathrm{C}_{7} \mathrm{H}_{16}$ & $\mathrm{C}_{7} \mathrm{H}_{16}$ & $\mathrm{O}_{2}$ & $\mathrm{O}_{2}$ & $\mathrm{O}_{2}$ & $\mathrm{O}_{2}$ \\
Species 1 & $\mathrm{N}_{2}$ & $\mathrm{~N}_{2}$ & $\mathrm{~N}_{2}$ & $\mathrm{~N}_{2}$ & $\mathrm{H}_{2}$ & $\mathrm{H}_{2}$ & $\mathrm{H}_{2}$ & $\mathrm{He}$ \\
$T_{2} ; T_{1}(\mathrm{~K})$ & $600 ; 1000$ & $600 ; 1000$ & $600 ; 1000$ & $600 ; 1000$ & $400 ; 600$ & $400 ; 600$ & $235 ; 287$ & $235 ; 287$ \\
$\rho_{2} / \rho_{1}$ & 12.88 & 12.88 & 12.88 & 12.88 & 24.40 & 24.40 & 24.51 & 12.17 \\
$p_{0}(\mathrm{~atm})$ & 60 & 60 & 60 & 60 & 100 & 100 & 100 & 100 \\
$R e_{0}$ & 400 & 500 & 600 & 800 & 750 & 550 & 500 & 600 \\
$\lambda_{1} / \delta_{\omega, 0}$ & 7.29 & 7.29 & 7.29 & 4.57 & 7.29 & 10.35 & 10.61 & 9.31 \\
$L_{1}(\mathrm{~m})$ & 0.200 & 0.200 & 0.200 & 0.125 & 0.200 & 0.284 & 0.284 & 0.255 \\
$L_{2}(\mathrm{~m})$ & 0.232 & 0.232 & 0.232 & 0.148 & 0.200 & 0.284 & 0.284 & 0.255 \\
$L_{3}(\mathrm{~m})$ & 0.120 & 0.120 & 0.120 & 0.075 & 0.120 & 0.170 & 0.170 & 0.153 \\
$\Delta x\left(10^{-4} \mathrm{~m}\right)$ & 10.71 & 8.36 & 6.97 & 5.23 & 5.77 & 8.19 & 8.39 & 7.36 \\
$F_{2 D} ; F_{3 D}$ & $0.1 ; 0.05$ & $0.1 ; 0.05$ & $0.1 ; 0.05$ & $0.1 ; 0.05$ & $0.1 ; 0.05$ & $0.1 ; 0.025$ & $0.1 ; 0.025$ & $0.05 ; 0.0125$ \\
$t_{t r}^{*}$ & 150 & 155 & 135 & 100 & 150 & 270 & 290 & 220 \\
$R e_{m, t r}$ & 972 & 1250 & 1452 & 1258 & 1507 & 1907 & 1772 & 2004
\end{tabular}

TABLE 2. Simulation parameters for supercritical temporal mixing layer database. $\lambda_{1}$ is the streamwise perturbation wavelength and $F_{2 D}$ and $F_{3 D}$ are the streamwise and spanwise perturbation amplitudes. All simulations have $M_{c .0}=0.4, L_{1}=4 \lambda_{1}$ and $L_{3}=0.6 L_{1}$. $\Delta x=\max \left(\Delta x_{1}, \Delta x_{2}, \Delta x_{3}\right)$.

to accommodate four wavelengths in the streamwise and spanwise directions, and the evolution of the layer is meant to encompass roll-up and two pairings of the four initial spanwise vortices into an ultimate vortex.

The boundary conditions are periodic in the streamwise and spanwise directions, and of outflow type for real gas in the cross-stream direction, as derived by Okong'o $\&$ Bellan (2002b). The outflow type conditions are essential to maintain numerical stability since the initial perturbation causes large pressure waves that must be allowed out of the domain with minimal reflection.

\subsection{Equation of state}

The pressure is calculated from the well-known Peng-Robinson (PR) EOS, given $T$ and the PR molar volume $\left(v_{P R}\right)$, as

$$
p=\frac{R_{u} T}{\left(v_{P R}-b_{m}\right)}-\frac{a_{m}}{\left(v_{P R}^{2}+2 b_{m} v_{P R}-b_{m}^{2}\right)},
$$

where $a_{m}$ and $b_{m}$ are functions of $T$ and $X_{\alpha}$ whose mathematical form is given in detail in Miller et al. (2001) and Okong'o et al. (2002). At high pressures, $v_{P R}$ may differ significantly from the actual molar volume $v$ (Prausnitz et al. 1986). Both $v_{P R}$ and the volume shift $\left(v_{S}=v-v_{P R}\right)$ can be calculated from the PR EOS given $p, T$ and $X_{\alpha}$ (Harstad, Miller \& Bellan 1997), although for the HN system $v_{S}$ is negligible. All thermodynamic quantities, including $\alpha_{D}, h, C_{p}=(\partial h / \partial T)_{p, X}$ and the speed of sound $\left(a_{s}\right)$, are calculated from the EOS using standard thermodynamic relations (Miller et al. 2001; Okong'o \& Bellan 2002a; Okong'o et al. 2002). By definition, $Z=p /\left(\rho T R_{u} / m\right)$ is the compression factor indicating departures from perfect-gas $(Z=1)$ behaviour. The implementation of the EOS to calculate $p$ and $T$ from $\rho, e$ and $Y_{\alpha}$ uses an iterative scheme (Okong'o et al. 2002) for $\mathrm{OH}$ and $\mathrm{OHe}$, and an energy fit (Okong'o \& Bellan 2002a) for HN. Since for a binary mixture the real-gas EOS is four-dimensional, as the variables are $\left(p, T, v, X_{2}\right)$, a plot of the EOS is not feasible; at fixed $v$, an example of a three-dimensional plot is shown in Prausnitz et al. (1986) (figure 10-6). 


$\begin{array}{lccc}\text { System } & \mathrm{C}_{7} \mathrm{H}_{16} / \mathrm{N}_{2} & \mathrm{O}_{2} / \mathrm{H}_{2} & \mathrm{O}_{2} / \mathrm{He} \\ \mu=\mu_{R}\left(T / T_{R}\right)^{n} & n=0.7 & n=0.75 & n=0.59 \\ S c \equiv \mu /\left(\rho \alpha_{D} D\right) & 1.5-Y_{2} & {\left[\left(1.334-0.668 Y_{2}-0.186 Y_{2}^{2}-0.268 Y_{2}^{6}\right)\right]} & \text { Eq. (A2) } \\ & \times\left[1+(88.6 / T)^{1.5}\right] & \\ P r \equiv \mu C_{p} /(m \lambda) & 0.5 S c / \exp \left(-1.5 Y_{2}\right) & 1.335 / T^{0.1} & \text { Eq. (A 1) } \\ \alpha_{I K} \text { or } \alpha_{B K} & \alpha_{I K}=0.1 & \alpha_{B K}=0.2 & \alpha_{B K}=0.25 \\ T \text { Range } & 500-1100 \mathrm{~K} & 200-800 \mathrm{~K} & 100-900 \mathrm{~K} \\ p \text { Range } & 40-80 \mathrm{~atm} & \sim 100 \mathrm{~atm} & \sim 100 \mathrm{~atm}\end{array}$

TABLE 3. Transport properties for binary mixtures. $T_{R}=\left(T_{1}+T_{2}\right) / 2, T$ in Kelvin. $\alpha_{I K}$ from Harstad \& Bellan (2000), and $\alpha_{B K}$ from Harstad \& Bellan (1998, 2001).

\subsection{Transport coefficients}

The viscosity, the Schmidt number $\left(S c=\mu /\left(\rho \alpha_{D} D\right)\right)$ and the Prandtl number $(\operatorname{Pr}=$ $\left.\mu C_{p} /(m \lambda)\right)$ were calculated from high-pressure single-species transport properties using mixing rules, as in Harstad \& Bellan (1998). The calculated values were correlated, as summarized in table 3 (see also Appendix A for $\mathrm{O}_{2} / \mathrm{He}$ ), and these correlations are then used to compute the transport properties $\mu, D$ and $\lambda$. The relationship between $\alpha_{B K}$ and $\alpha_{I K}$ stated in equation (2.15) means that either one can be specified, and the other then calculated.

\subsection{Relevance of the database to turbulent flow modelling}

The database used for the a priori analysis is summarized in table 2, including the transitional time $t_{t r}^{*}=t_{t r} \Delta U_{0} / \delta_{\omega, 0}$ and the value of the momentum-thicknessbased Reynolds number, $R e_{m}=R e_{0} \delta_{m} / \delta_{\omega, 0}$ at transition. Both $t_{t r}$ and $\delta_{m}$ are defined in Okong'o \& Bellan $(2002 a) ; t_{t r}$ is the time at which the one-dimensional velocityfluctuation-based energy spectra become smooth, except for the forcing frequency, and $\delta_{m}$ is the momentum thickness. The grid spacing is uniform with $\Delta x_{1} \simeq \Delta x_{2} \simeq \Delta x_{3}$, with the notation $\Delta x=\max \left\{\Delta x_{i}\right\}$. The differing thermodynamics of the various species systems preclude matching of the initial density stratification $\left(\rho_{2} / \rho_{1}\right)$ or of the transitional momentum thickness (Okong'o et al. 2002; Okong'o \& Bellan 2003) within the regimes of practical interest, since there is not a simple relationship between the initial conditions and the transitional state. As a result, the $\mathrm{O}_{2}$ layers exhibited higher $R e_{m}$ values at the transitional state, $R e_{m, t r}$, with the OHe layer having the highest value of 2004. Results from the database can be compared either for same species but at different $R e_{m, t r}$ (HN layers), or same species and same $R e_{m, t r}$ (HN500, HN800), or different species and same $R e_{m, t r}((\mathrm{HN} 600, \mathrm{OH} 750)$ and (OH550, OHe600)).

The most prominent feature of the transitional states are HDGM regions that populate the entire mixing layer. Animations of the layers' evolution from the initial state to transition showed that the HDGM regions were formed as a combination of the species mixing and of the distortion of the initial density gradient boundary. While the HN layers had lower initial $\rho_{2} / \rho_{1}$, they had higher densitygradient magnitude $|\nabla \rho| \delta_{\omega, 0} /\left(\rho_{2}-\rho_{1}\right)$ at the transitional state, due to their higher mixture non-ideality (Okong'o \& Bellan 2004a) which impeded molecular mixing. Experimental evidence exists indicating that the HDGM regions are not a peculiar aspect of the transitional state, but persist with similar or even larger gradient magnitudes at fully turbulent conditions. Supporting this argument, temporally accurate measurements of the density gradients are shown in figure 2 which displays results originated from planar laser-induced fluorescence found from injection of liquid 

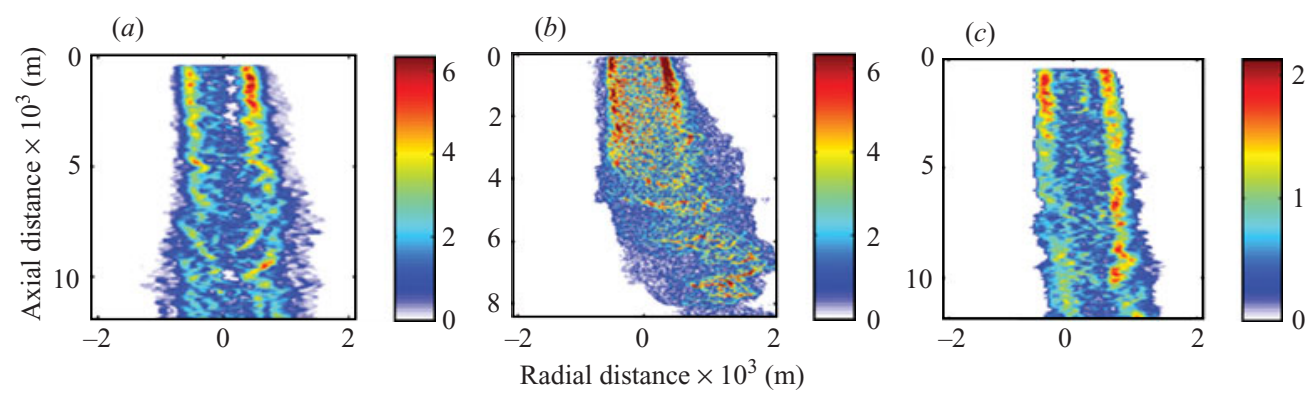

FIGURE 2. Density gradient (colour bar shows $\nabla \rho \times 10^{-6} \mathrm{~kg} \mathrm{~m}^{-4}$ ) from the experiments of Polikhov \& Segal (2007) where a fluoreketone $\left(T_{c}=441 \mathrm{~K}, p_{c}=1.87 \mathrm{MPa}\right)$ jet of $0.84 \times 10^{-3} \mathrm{~m}$ diameter is injected into a coaxial flow of $\mathrm{N}_{2}$. $(a) p_{c h}=2.08 \mathrm{MPa}, T_{c h}=394 \mathrm{~K}, T_{j e t}=291 \mathrm{~K}$ and $R e_{j e t}=29200$. (b) $p_{c h}=3.14 \mathrm{MPa}, T_{c h}=509 \mathrm{~K}, T_{j e t}=305 \mathrm{~K}$ and $R e_{j e t}=58600$. (c) $p_{c h}=3.97 \mathrm{MPa}, T_{c h}=422 \mathrm{~K}, T_{j e t}=488 \mathrm{~K}$ and $R e_{j e t}=57500$. Subscripts $c h$ and $j e t$ denote the chamber and the jet. Information and plots courtesy of C. Segal (2006, personal communication).

dodecafluoro-2-methylpentan-3-pentanone in a chamber filled with $\mathrm{N}_{2}$. In these experiments, the chamber has a square cross-section of $0.025 \mathrm{~m}$ side and $0.19 \mathrm{~m}$ length, and can be pressurized up to $7 \mathrm{MPa}$ and heated to more than $500 \mathrm{~K}$. Optical access is available for an axial distance larger than $0.1 \mathrm{~m}$ through windows flush with the chamber walls. The chamber walls and a constant axial flow of $\mathrm{N}_{2}$ are heated until the experimental temperature and the pressure are reached. The liquid is then injected through an electric heater into the chamber through a $0.84 \times 10^{-3} \mathrm{~m}$ diameter orifice. The goal was to inject into a uniform flow of $\mathrm{N}_{2}$, although this goal was not always achieved as evident in figure $2(b)$ and to a smaller extent in figure $2(c)$. A thinner than $0.3 \times 10^{-3} \mathrm{~m}$ laser sheet at $355 \times 10^{-9} \mathrm{~m}$ is sent through the jet and the induced fluorescence at $420 \pm 10 \times 10^{-9} \mathrm{~m}$ is collected on a CCD camera.

While the data are very rich and discussed in detail in Polikhov \& Segal (2007), the interest here is only in whether large density gradients are observed under supercritical conditions at higher $R e$ values than those generated in DNS. Three experimental conditions are shown in figure 2, where in all experiments $R e=O\left(10^{4}\right)$ at the injection location and the pressure was supercritical with respect to the injected liquid (see figure 2 caption). The reduced thermodynamic conditions $\left(p_{r}=p / p_{c}\right.$, $\left.T_{r}=T / T_{c}\right)$ of figure 2(c) for the liquid $\left(p_{r}=2.12, T_{r}=1.11\right)$ are similar to those for heptane $\left(p_{r}=2.19, T_{r}=1.11\right)$ in $\mathrm{HN}$ simulations. The maximum density gradient value, $O\left(10^{6}\right) \mathrm{kg} \mathrm{m}^{-4}$, exceeds by an order of magnitude that found in the DNS for the conditions listed in table 2 .

Even larger $R e$ values at the injection location, $R e=O\left(10^{5}\right)$, are used in experiments performed with a $\mathrm{N}_{2}$ free jet either without coaxial hydrogen injection (Oschwald \& Schik 1999) or with hydrogen injection (Oschwald et al. 1999); the experimental conditions are listed in table 4 . Illustrated in figure 3 is the radial density gradient experimentally obtained in these fully turbulent jets at fixed axial positions. In the measurements, Raman signals were recorded during $1 \mathrm{~s}$ and therefore the data are temporally averaged, meaning that gradients are smoothed due to turbulent fluctuations; the gradient values shown thus represent a lower bound of instantaneous density gradients such as computed from DNS. The prominent experimental densitygradient peaks are $O\left(10^{5}\right) \mathrm{kg} \mathrm{m}^{-4}$ and minor peaks with $O\left(10^{4}\right) \mathrm{kg} \mathrm{m}^{-4}$ populate the entire extent of the flow. These experimental density gradients have the same 


$\begin{array}{lccccccccc}\text { Figure } & p_{c h} & T_{c h} & T_{i n j, N_{2}} & T_{i n j, H_{2}} & \rho_{j e t, N_{2}} / \rho_{c h} & \rho_{j e t, N_{2}} / \rho_{j e t, H_{2}} & \operatorname{Re}_{N_{2}} & \operatorname{Re}_{H_{2}} & x_{\text {meas }} \\ 3(a) & 4 & 298 & 140 & - & 3.34 & - & 1.15 \times 10^{5} & - & 2 \\ 3(b) & 4 & 298 & 118 & - & 12.50 & - & 1.26 \times 10^{5} & - & 16 \\ 3(c) & 4 & 288 & 140 & 270 & - & 43.06 & 3.14 \times 10^{4} & 1.01 \times 10^{4} & 10 \\ 3(d) & 4 & 288 & 118 & 270 & - & 166.68 & 1.22 \times 10^{5} & 2.02 \times 10^{4} & 40\end{array}$

TABLE 4. Experimental conditions corresponding to Oschwald \& Schik (1999) and Oschwald et al. (1999). The pressure is in $\mathrm{MPa}$, all temperatures are in $\mathrm{K}$ and distances in $10^{-3} \mathrm{~m} . x$ is the axial distance from the injection location. Subscripts $c h$ and jet denote the chamber and jet conditions, respectively; inj labels the injection location; and meas denotes the measurement location.
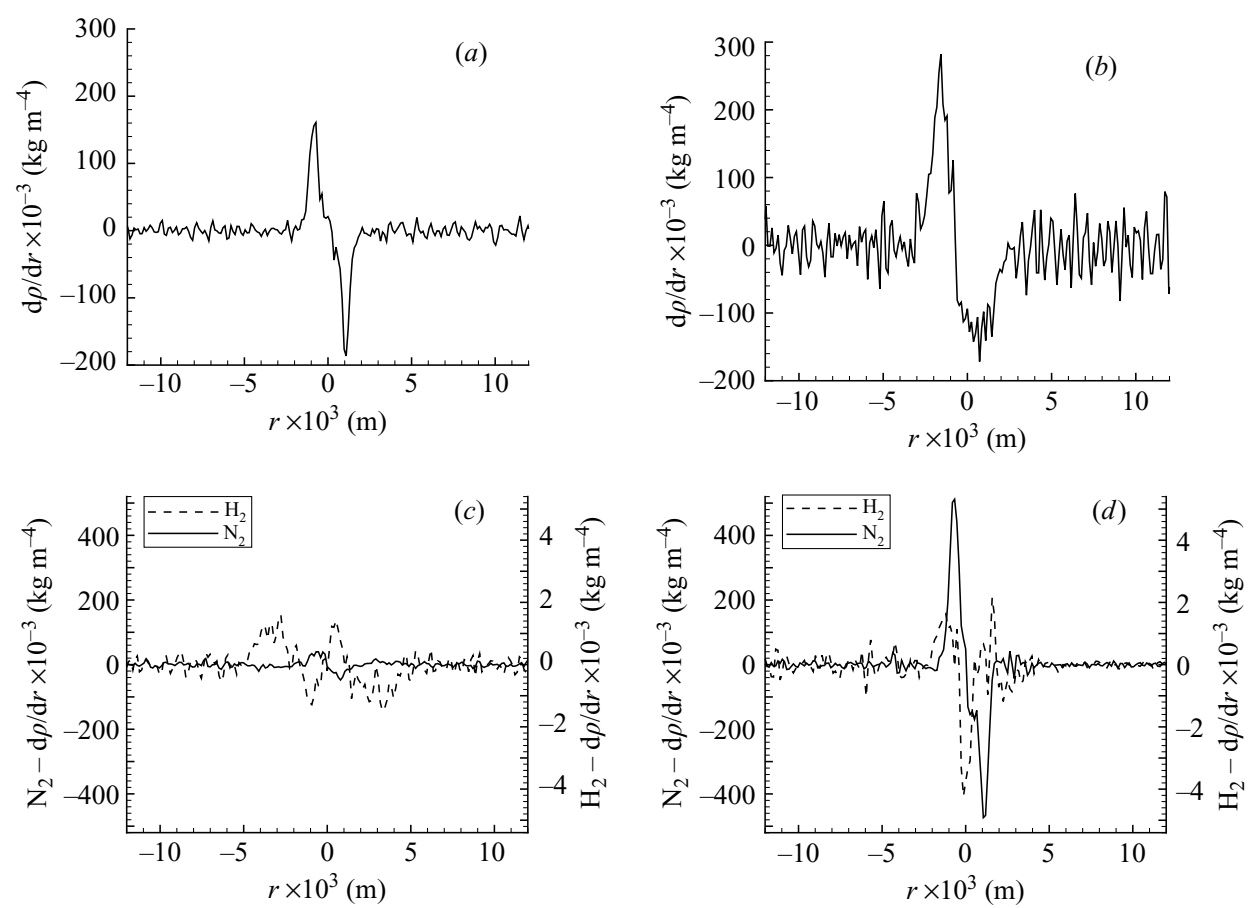

FiguRE 3. Radial density gradient from the nitrogen injection experiments of Oschwald \& Schik (1999) $(a, b)$, and the nitrogen/coaxial-hydrogen injection experiments of Oschwald et al. (1999) $(c, d)$. $(a)$ and $(b)$ correspond to figures 5 and 6 of Oschwald \& Schik (1999), respectively. $(c)$ and $(d)$ correspond to figures 9 and 10 of Oschwald et al. (1999), respectively. Information on detailed experimental conditions is in table 4. Data and details not available in the experimental papers were obtained courtesy of M. Oschwald (2006, personal communication).

magnitude as those from the DNS database (e.g. Okong'o \& Bellan 2002a), $O\left(10^{4}\right)-O\left(10^{5}\right) \mathrm{kg} \mathrm{m}^{-4}$. Thus, the experimental observations show that under supercritical conditions, turbulence $\left(R e=O\left(10^{4}\right)-O\left(10^{5}\right)\right)$ does not smear the HDGM regions which remain a standing, specific character of the flow. The role of these HDGM regions was discussed by Miller et al. (2001) and Bellan (2006), particularly the redistribution of turbulent energy from the normal direction to the HDGM (at each point) to the tangential direction (at the same point) found in the experiments of Hannoun, Fernando \& List (1988) at sharp density boundaries. 
Unless one can capture this local aspect of the flow, it is very unlikely that mixing and combustion could be faithfully simulated in gas-turbine, diesel or liquid rocket engines. Because the HDGM regions are thinner for the HN layers, and since the appropriate LES resolution $\left(\Delta x_{L E S}\right)$ depends on the gradients of the filtered flow field, which in turn depends on the filter width $\bar{\Delta}$, the implication is that HN LES may require higher resolution relative to DNS (i.e. less grid coarsening, smaller $\bar{\Delta} / \Delta x_{D N S}$ and $\left.\Delta x_{L E S} / \Delta x_{D N S}\right)$ than LES for the other species systems considered.

Further supporting the relevance to LES of the database transitional states is the significant portion of the domain turbulent kinetic energy (TKE) that resides in the subgrid scales. At $\bar{\Delta} / \Delta x=4$, and for fluctuations based on the filtered velocity, up to $9.03 \%$ of the TKE resides in the small scales; the equivalent value for fluctuations based on the Favre-averaged velocity is $10.63 \%$. When the filter size increases by a factor of 2 to $\bar{\Delta} / \Delta x=8$, the corresponding fraction of the TKE in the small scales increases by more than a factor of 2.5 and at given $\bar{\Delta}$, the TKE portion residing in the subgrid scales is smaller with increasing $R e_{0}$.

\section{Results}

A cubic top-hat filter is used for the flow field, for which the filtered value is simply the volume average. The filter width used is $\bar{\Delta}$, with $\bar{\Delta} / \Delta x=4$ or 8 . Further filtering is performed at the test-filter width $\hat{\Delta}$, with $\hat{\Delta} / \bar{\Delta}=1$ or 2 . All calculations are performed on the DNS grid. The analysis is carried out at the transitional states listed in table 2.

\subsection{LES assumptions}

\subsubsection{Evaluation of the LES assumptions}

Following a protocol previously used for an atmospheric two-phase mixing layer (Okong'o \& Bellan 2004b), the following LES assumptions are evaluated, in the spirit of simplifying equations (2.28)-(2.30):

$$
\begin{gathered}
\tilde{e}=e(\bar{\phi}), \quad \bar{T}=T(\bar{\phi}), \quad \widetilde{T}=T(\bar{\phi}), \quad \bar{p}=p(\bar{\phi}), \quad \tilde{h}=h(\bar{\phi}), \\
\bar{\sigma}_{i j}=\sigma_{i j}(\bar{\phi}), \quad \bar{j}_{\alpha j}=j_{\alpha j}(\bar{\phi}), \quad \bar{q}_{I K j}=q_{I K j}(\bar{\phi}), \quad \overline{u_{i} \sigma_{i j}}=\widetilde{u}_{i} \sigma_{i j}(\bar{\phi}), \\
\bar{\rho} \kappa_{j}=\frac{1}{2}\left(\overline{\rho u_{i} u_{i} u_{j}}-\overline{\rho u_{i} u_{i}} \widetilde{u}_{j}\right)=\bar{\rho} \tau_{i j} \widetilde{u}_{i} .
\end{gathered}
$$

Also, consider the quantities

$$
\left.\begin{array}{c}
\frac{\partial}{\partial x_{i}}[\bar{p}-p(\bar{\phi})], \quad \frac{\partial}{\partial x_{j}}\left[\bar{\sigma}_{i j}-\sigma_{i j}(\bar{\phi})\right], \\
\frac{\partial}{\partial x_{j}}\left\{[\bar{p}-p(\bar{\phi})] \tilde{u}_{j}\right\}, \quad \frac{\partial}{\partial x_{j}}\left[\bar{q}_{I K j}-q_{I K j}(\bar{\phi})\right], \quad \frac{\partial}{\partial x_{j}}\left[\bar{\sigma}_{i j} u_{i}-\sigma_{i j}(\bar{\phi}) \tilde{u}_{i}\right], \\
\frac{\partial}{\partial x_{j}}\left[\bar{j}_{\alpha j}-j_{\alpha j}(\bar{\phi})\right],
\end{array}\right\}
$$

for $(i, j) \in[1,3]$; if these quantities are much smaller, according to a selected norm, than the leading terms in equations (2.28)-(2.30), then they may be neglected. Vreman, Guerts \& Kuerten (1995) utilized the L2 norm to perform such a comparison. The selected norm is here the domain r.m.s. (square root of the L2 norm) because it quantifies each term's activity.

The LES assumptions equations (4.1)-(4.3) were computed both in terms of correlations and slopes from a least-squares fit of the model (right-hand side) to the terms (left-hand side) at two filter sizes, $\bar{\Delta} / \Delta x=4$ and 8 . Correlations between the 


\begin{tabular}{|c|c|c|c|c|c|c|}
\hline \multirow[b]{2}{*}{$x_{1}$-momentum } & \multicolumn{3}{|c|}{$\bar{\Delta}=4 \Delta x$} & \multicolumn{3}{|c|}{$\bar{\Delta}=8 \Delta x$} \\
\hline & HN600 & $\mathrm{OH} 750$ & OHe600 & HN600 & $\mathrm{OH} 750$ & OHe600 \\
\hline$\frac{\partial}{\partial x_{j}}\left(\bar{\rho} \tilde{u}_{1} \tilde{u}_{j}\right)$ & 24.5 & 28.7 & 41.5 & 19.9 & 27.0 & 35.0 \\
\hline$\frac{\partial}{\partial x_{1}}[p(\bar{\phi})]$ & 12.1 & 18.5 & 16.3 & 13.4 & 18.3 & 14.6 \\
\hline$\frac{\partial}{\partial x_{j}}\left[\sigma_{1 j}(\bar{\phi})\right]$ & 1.17 & 3.18 & 2.25 & 0.752 & 2.69 & 1.55 \\
\hline$\frac{\partial}{\partial x_{j}}\left(\bar{\rho} \tau_{1 j}\right)$ & 1.49 & 0.890 & 1.82 & 2.48 & 2.11 & 3.29 \\
\hline$\frac{\partial}{\partial x_{1}}[\Delta(\bar{p})]$ & 6.87 & 0.308 & 2.09 & 10.5 & 0.640 & 3.16 \\
\hline$\frac{\partial}{\partial x_{j}}\left[\Delta\left(\bar{\sigma}_{1 j}\right)\right]$ & 0.312 & 0.789 & 0.503 & 0.328 & 1.37 & 0.604 \\
\hline & & $\Delta($ & $f(\phi)$ & & & \\
\hline
\end{tabular}

TABle 5. Magnitude (r.m.s.) of terms in the LES equations at $t_{t r}^{*}$. Units are $10^{6} \mathrm{~N} \mathrm{~m}^{-3}$.

models and terms (not shown) were excellent (typically above $98 \%$ ), being somewhat lower for the larger filter width. Correspondingly, compared to the values at the smaller $\bar{\Delta} / \Delta x$, the slopes (not shown) also exhibit greater deviation from the ideal value of unity at the larger filter width. The thermodynamic assumptions (equation (4.1)) have less than $1 \%$ error on all the flow fields. The assumptions for the viscous, heat and species-mass fluxes are almost as accurate, with errors of about $4 \%$. The model for the triple correlation (equation (4.3)) appears to be the least accurate assumption, with errors of up to $10 \%$. Therefore, it would appear that the filtered thermodynamic quantities (internal energy, temperature, pressure and enthalpy, but not necessarily their gradients) and the filtered viscous, heat and species-mass fluxes (but not necessarily their gradients) can be adequately modelled from the filtered flow field. The same results regarding the filtered quantities were previously obtained in an atmosphericpressure perfect-gas gaseous mixing layer (Okong'o \& Bellan 2004b), although here the species-mass and heat fluxes have a much more complicated functional form (equations (2.10) and (2.11)), including Soret and Dufour (thermal diffusion) effects.

To test whether the quantities listed in (4.4)-(4.5) are much smaller than the leading terms in equations (2.28)-(2.30), the domain r.m.s. of terms in the conservation equations were computed and examples are shown in tables 5-9. All calculations were performed on the DNS grid. Although the flow evolution will be different in LES and DNS, it seems reasonable to assume that larger-magnitude unclosed terms in the filtered DNS equations would require a more accurate model in LES. The examples chosen in tables 5-9 are typical of $\mathrm{HN}$ and $\mathrm{OH}$ simulations, and also allow comparison of $\mathrm{HN}$ and $\mathrm{OH}$ simulations with similar $R e_{m, t r}$; finally the $\mathrm{OHe}$ simulation has the largest $R e_{m, t r}$. Also, the HN flows exhibit strong departures from perfect gas and mixture ideality (Okong'o \& Bellan 2002a); the OHe flow is close to an ideal mixture but displays moderate departures from perfect gas (not shown), and the $\mathrm{OH}$ flows are nearly perfect gases and ideal mixtures for the conditions of these simulations (Okong'o et al. 2002).

Considering the momentum equation (tables 5-7), similar to atmospheric-pressure gaseous-flow findings (Okong'o \& Bellan 2004b), the convective and resolved pressure 


\begin{tabular}{cccccccc} 
& \multicolumn{3}{c}{$\bar{\Delta}=4 \Delta x$} & & \multicolumn{3}{c}{$\bar{\Delta}=8 \Delta x$} \\
\cline { 2 - 3 }$x_{2}$-momentum & HN600 & OH750 & OHe600 & & HN600 & OH750 & OHe600 \\
$\frac{\partial}{\partial x_{j}}\left(\bar{\rho} \tilde{u}_{2} \tilde{u}_{j}\right)$ & 17.4 & 21.2 & 31.6 & & 13.9 & 19.8 & 26.7 \\
$\frac{\partial}{\partial x_{2}}[p(\bar{\phi})]$ & 15.6 & 13.8 & 18.8 & & 19.0 & 13.3 & 16.5 \\
$\frac{\partial}{\partial x_{j}}\left[\sigma_{2 j}(\bar{\phi})\right]$ & 0.865 & 1.74 & 1.70 & & 0.558 & 1.41 & 1.17 \\
$\frac{\partial}{\partial x_{j}}\left(\bar{\rho} \tau_{2 j}\right)$ & 1.38 & 0.744 & 1.85 & & 2.52 & 1.81 & 3.58 \\
$\frac{\partial}{\partial x_{2}}[\Delta(\bar{p})]$ & 10.6 & 0.640 & 2.97 & & 16.7 & 1.31 & 4.79 \\
$\frac{\partial}{\partial x_{j}}\left[\Delta\left(\bar{\sigma}_{2 j}\right)\right]$ & 0.185 & 0.300 & 0.331 & & 0.176 & 0.497 & 0.335 \\
& & $\Delta(\bar{f}) \equiv \overline{f(\phi)}-f(\bar{\phi})$ & & &
\end{tabular}

TABLE 6. Magnitude (r.m.s.) of terms in the LES equations at $t_{t r}^{*}$. Units are $10^{6} \mathrm{~N} \mathrm{~m}^{-3}$.

\begin{tabular}{cccccccc} 
& \multicolumn{3}{c}{$\bar{\Delta}=4 \Delta x$} & & \multicolumn{3}{c}{$\bar{\Delta}=8 \Delta x$} \\
\cline { 2 - 3 } \cline { 7 - 8 }$x_{3}$-momentum & HN600 & OH750 & OHe600 & & HN600 & OH750 & OHe600 \\
$\frac{\partial}{\partial x_{j}}\left(\bar{\rho} \tilde{u}_{3} \tilde{u}_{j}\right)$ & 16.0 & 9.00 & 31.7 & & 12.2 & 7.44 & 26.4 \\
$\frac{\partial}{\partial x_{3}}[p(\bar{\phi})]$ & 14.5 & 6.08 & 19.7 & & 16.3 & 5.00 & 17.2 \\
$\frac{\partial}{\partial x_{j}}\left[\sigma_{3 j}(\bar{\phi})\right]$ & 0.730 & 0.739 & 1.83 & & 0.464 & 0.584 & 1.27 \\
$\frac{\partial}{\partial x_{j}}\left(\bar{\rho} \tau_{3 j}\right)$ & 1.23 & 0.367 & 1.90 & & 2.16 & 0.851 & 3.88 \\
$\frac{\partial}{\partial x_{3}}[\Delta(\bar{p})]$ & 10.4 & 0.839 & 2.97 & & 14.4 & 1.75 & 4.74 \\
$\frac{\partial}{\partial x_{j}}\left[\Delta\left(\bar{\sigma}_{3 j}\right)\right]$ & 0.124 & 0.106 & 0.301 & & 0.118 & 0.171 & 0.315 \\
& & $\Delta(\bar{f}) \equiv \overline{f(\phi)}-f(\bar{\phi})$ & & &
\end{tabular}

TABle 7. Magnitude (r.m.s.) of terms in the LES equations at $t_{t r}^{*}$. Units are $10^{6} \mathrm{~N} \mathrm{~m}^{-3}$.

terms are of leading order and the resolved stresses and subgrid flux term are approximately one order of magnitude smaller; unlike the atmospheric-pressure findings, $\nabla[\bar{p}-p(\bar{\phi})]$ is of same order as $\nabla(p(\bar{\phi}))$ for flows exhibiting strong departures from perfect-gas and mixture-ideality conditions (i.e. HN cases; Okong'o \& Bellan $2004 b$ ), whereas for near-perfect gas and ideal-mixture flows (i.e. OH cases; Okong'o et al. 2002; Okong'o \& Bellan 2003) the term is again one order of magnitude smaller than the leading terms, with the $\mathrm{OHe}$ case being intermediary between the HN and $\mathrm{OH}$ ones. Of note, is that for each simulation, both $\partial(p(\bar{\phi})) / \partial x_{j}$ and $\partial[\bar{p}-p(\bar{\phi})] / \partial x_{j}$ have similar values for all three components of the momentum equation, meaning that the flow is strongly three-dimensional, thus casting serious doubts on two-dimensional LES of supercritical flows (e.g. Zong et al. 2004; Oefelein 2005). 


\begin{tabular}{|c|c|c|c|c|c|c|}
\hline \multirow[b]{2}{*}{ Energy } & \multicolumn{3}{|c|}{$\bar{\Delta}=4 \Delta x$} & \multicolumn{3}{|c|}{$\bar{\Delta}=8 \Delta x$} \\
\hline & HN600 & OH750 & OHe600 & HN600 & $\mathrm{OH} 750$ & OHe600 \\
\hline$\frac{\partial}{\partial x_{j}}\left(\bar{\rho} \tilde{e}_{t} \tilde{u}_{j}\right)$ & 219 & 48.1 & 20.5 & 178 & 47.5 & 18.6 \\
\hline$\frac{\partial}{\partial x_{j}}\left[p(\bar{\phi}) \tilde{u}_{j}\right]$ & 4.08 & 19.8 & 8.77 & 4.21 & 19.7 & 8.71 \\
\hline$\frac{\partial}{\partial x_{j}}\left[q_{I K j}(\bar{\phi})\right]$ & 4.06 & 59.4 & 13.7 & 3.45 & 46.8 & 8.38 \\
\hline$\frac{\partial}{\partial x_{j}}\left[\sigma_{i j}(\bar{\phi}) \tilde{u}_{i}\right]$ & 0.164 & 1.51 & 0.579 & 0.109 & 1.40 & 0.425 \\
\hline$\frac{\partial}{\partial x_{j}}\left(\bar{\rho} \zeta_{j}\right)$ & 2.35 & 13.2 & 9.06 & 2.99 & 30.9 & 14.6 \\
\hline$\frac{\partial}{\partial x_{j}}\left(\bar{\rho} \tau_{i j} \tilde{u}_{i}\right)$ & 0.158 & 0.303 & 0.357 & 0.253 & 0.682 & 0.666 \\
\hline$\frac{\partial}{\partial x_{j}}\left(\bar{\rho} \kappa_{j}-\bar{\rho} \tau_{i j} \widetilde{u}_{i}\right)$ & 0.0257 & 0.0170 & 0.0474 & 0.0659 & 0.0938 & 0.155 \\
\hline$\frac{\partial}{\partial x_{j}}\left\{\Delta(\bar{p}) \tilde{u}_{j}\right\}$ & 0.469 & 0.0717 & 0.215 & 0.826 & 0.155 & 0.378 \\
\hline$\frac{\partial}{\partial x_{j}}\left[\Delta\left(\bar{q}_{I K j}\right)\right]$ & 1.82 & 11.3 & 3.56 & 2.45 & 19.8 & 3.35 \\
\hline$\frac{\partial}{\partial x_{j}}\left[\Delta\left(\overline{\sigma_{i j} u_{i}}\right)\right]$ & 0.0601 & 0.494 & 0.179 & 0.0639 & 0.865 & 0.228 \\
\hline & & $\Delta(\bar{f})$ & $\overline{\phi)}-j$ & & & \\
\hline
\end{tabular}

TABLE 8. Magnitude (r.m.s.) of terms in the LES equations at $t_{t r}^{*}$. Units are $10^{9} \mathrm{~J} \mathrm{~m}^{-3} \mathrm{~s}^{-1}$.

\begin{tabular}{|c|c|c|c|c|c|c|}
\hline \multirow[b]{2}{*}{ Species } & \multicolumn{3}{|c|}{$\bar{\Delta}=4 \Delta x$} & \multicolumn{3}{|c|}{$\bar{\Delta}=8 \Delta x$} \\
\hline & HN600 & $\mathrm{OH} 750$ & OHe600 & HN600 & OH750 & OHe600 \\
\hline$\frac{\partial}{\partial x_{j}}\left(\bar{\rho} \tilde{Y}_{2} \tilde{u}_{j}\right)$ & 28.9 & 13.0 & 27.7 & 23.4 & 11.9 & 23.7 \\
\hline$\frac{\partial}{\partial x_{j}}\left[j_{2 j}(\bar{\phi})\right]$ & 1.17 & 0.706 & 0.985 & 0.808 & 0.556 & 0.600 \\
\hline$\frac{\partial}{\partial x_{j}}\left(\bar{\rho} \eta_{2 j}\right)$ & 0.755 & 0.157 & 0.647 & 1.20 & 0.365 & 1.05 \\
\hline$\frac{d}{\partial x_{j}}\left[\Delta\left(\overline{j_{2 j}}\right)\right]$ & 0.469 & 0.129 & 0.237 & 0.515 & 0.227 & 0.222 \\
\hline
\end{tabular}

TABle 9. Magnitude (r.m.s.) of terms in the LES equations at $t_{t r}^{*}$. Units are $10^{4} \mathrm{~kg} \mathrm{~m}^{-3} \mathrm{~s}^{-1}$.

For the energy equation (table 8), in HN simulations, the convective term leads by about 2 orders of magnitude the pressure work, heat flux, subgrid enthalpy and $\partial\left[\bar{q}_{I K j}-q_{I K j}(\bar{\phi})\right] / \partial x_{j}$; a third category of even smaller terms is that of the stress work, the triple correlation, $\partial\left\{[\bar{p}-p(\bar{\phi})] \tilde{u}_{j}\right\} / \partial x_{j}$ and $\partial\left[\overline{\sigma_{i j} u_{i}}-\sigma_{i j}(\bar{\phi}) \tilde{u}_{i}\right] / \partial x_{j}$; the smallest term is that of the LES assumption on the triple correlation. This ordering of terms is unlike that for atmospheric-pressure gaseous flows (Okong'o \& Bellan 2004b) or that for the $\mathrm{OH}$ cases where the convective, pressure work, resolved heat flux and subgrid heat flux are leading terms, with the additional contribution of $\partial\left[\bar{q}_{I K j}-q_{I K j}(\bar{\phi})\right] / \partial x_{j}$ 
compared to atmospheric gaseous flows; the stress work is one order of magnitude smaller than the leading terms, followed by the triple correlation, $\partial\left\{[\bar{p}-p(\bar{\phi})] \tilde{u}_{j}\right\} / \partial x_{j}$ and the assumption of the triple correlation $\partial\left(\bar{\rho} \kappa_{j}-\bar{\rho} \tau_{i j} \widetilde{u}_{i}\right) / \partial x_{j}$. As for the OHe case, of leading order are the convective, heat-flux, subgrid-heat-flux and pressure-work terms, with all other terms being one to three orders of magnitude smaller than the leading-order terms.

For the species equations, for all species pairs, the convective term dominates all other terms by at least one order of magnitude. Comparing the results at $\bar{\Delta} / \Delta x=4$ and 8 , the relative magnitude of the terms' activity in the LES equations is filter-size independent.

The mathematical rationale for justifying the existence of new LES-equations terms with respect to atmospheric flow situations is directly related to the strongly nonlinear aspect of the PR EOS. In fact, filtering the DNS equations includes filtering of the EOS, which due to its nonlinear aspect yields SGS terms that must be modelled. That is, the filtered solution does not satisfy the original EOS. Because the perfect-gas EOS has much weaker nonlinearities, the LES equations did not include additional terms for atmospheric-pressure gas flows (Okong'o \& Bellan 2004b) for which the perfect-gas EOS is valid. The strong nonlinearities of the PR EOS induce large changes in $p$ even when the changes in the other thermodynamic variables are small; this is the essence of a strong nonlinearity. Since LES is meant to be accurate and computationally efficient, computational efficiency dictates that because $T$ is found (from a fit or an iterative solution) as a function of $e$ and $\rho$ that is calculated from the EOS (Miller et al. 2001; Okong'o \& Bellan 2002a), it is desirable to retain the ability to use the same PR EOS for the filtered solution rather than modelling the complicated unknown terms in the filtered PR EOS. However, to satisfy computational efficiency without sacrificing accuracy, one must account for the EOS-induced new terms in the differential conservation equations.

Thus, it appears that effort must be devoted to modelling one term that has never been included in LES momentum equations, namely $\nabla[\bar{p}-p(\bar{\phi})]$, which is third in magnitude for $\mathrm{HN}$ cases. Also, in the $\mathrm{OH}$ cases $\nabla \cdot\left[\boldsymbol{q}_{I K}-\boldsymbol{q}_{I K}(\bar{\phi})\right]$ is of same order of magnitude as the leading term in the energy equation, and thus deserves similar attention. With these two exceptions, all other than the resolved terms and SGS fluxes are neglected, based on their smaller magnitude compared to the leading-order terms.

Incorporating the validated LES assumptions, equations (2.27)-(2.30) become

$$
\begin{gathered}
\frac{\partial \bar{\rho}}{\partial t}+\frac{\partial \bar{\rho} \tilde{u}_{j}}{\partial x_{j}}=0 \\
\frac{\partial \bar{\rho} \tilde{u}_{i}}{\partial t}+\frac{\partial \bar{\rho} \tilde{u}_{i} \tilde{u}_{j}}{\partial x_{j}}=-\frac{\partial p(\bar{\phi})}{\partial x_{i}}+\frac{\partial \sigma_{i j}(\bar{\phi})}{\partial x_{j}}-\frac{\partial\left(\bar{\rho} \tau_{i j}\right)}{\partial x_{j}}-\frac{\partial[\bar{p}-p(\bar{\phi})]}{\partial x_{i}}, \\
\frac{\partial \bar{\rho} \tilde{e}_{t}}{\partial t}+\frac{\partial \tilde{\rho} \tilde{e}_{t} \tilde{u}_{j}}{\partial x_{j}}=-\frac{\partial p(\bar{\phi}) \tilde{u}_{j}}{\partial x_{j}}-\frac{\partial q_{I K j}(\bar{\phi})}{\partial x_{j}}+\frac{\partial \sigma_{i j}(\bar{\phi}) \tilde{u}_{i}}{\partial x_{j}} \\
-\frac{\partial\left(\bar{\rho} \zeta_{j}\right)}{\partial x_{j}}-\frac{\partial\left(\bar{\rho} \tau_{i j} \tilde{u}_{i}\right)}{\partial x_{j}}-\frac{\partial\left[\bar{q}_{I K j}-q_{I K j}(\bar{\phi})\right]}{\partial x_{j}}, \\
\frac{\partial \bar{\rho} \tilde{Y}_{\alpha}}{\partial t}+\frac{\partial \bar{\rho} \tilde{Y}_{\alpha} \tilde{u}_{j}}{\partial x_{j}}=-\frac{\partial j_{\alpha j}(\bar{\phi})}{\partial x_{j}}-\frac{\partial\left(\bar{\rho} \eta_{\alpha j}\right)}{\partial x_{j}} .
\end{gathered}
$$

\subsubsection{Modelling of $\nabla[\bar{p}-p(\bar{\phi})]$}

The driving idea for modelling $\nabla[\bar{p}-p(\bar{\phi})]$ is to expand the EOS equation (3.1) in a Taylor series. However, $p$ is available from the EOS as a function of the 
thermodynamic primitive variables in vector $\psi$, whereas the solution of the LES equations is computed for the thermodynamic conservative variables in vector $\phi$. Choosing the reference state $\bar{\phi}$, the filtered expansion is

$$
\overline{p(\phi)}=\overline{p(\bar{\phi})}+\overline{\left.\frac{\partial p}{\partial \phi_{m}}\right|_{\phi=\bar{\phi}}\left(\phi_{m}-\overline{\phi_{m}}\right)}+\overline{\left.\frac{1}{2} \frac{\partial^{2} p}{\partial \phi_{m} \partial \phi_{n}}\right|_{\phi=\bar{\phi}}\left(\phi_{m}-\overline{\phi_{m}}\right)\left(\phi_{n}-\overline{\phi_{n}}\right)}+O\left(\overline{\phi^{3}}\right),
$$

which, under the (non-rigorous) assumptions that

$$
\overline{p(\bar{\phi})}=p(\bar{\phi})
$$

that $\left(\partial p / \partial \phi_{m}\right)_{\phi=\bar{\phi}}$ and $\left(\partial^{2} p /\left(\partial \phi_{m} \partial \phi_{n}\right)\right)_{\phi=\bar{\phi}}$ can be removed from the filtering operation, and that the filter is a projection which implies that $\left(\overline{\phi_{m}}-\overline{\overline{\phi_{m}}}\right)=0$, leads to

$$
\overline{p(\phi)}=p(\bar{\phi})+\delta, \delta=\left.\frac{1}{2} \frac{\partial^{2} p}{\partial \phi_{m} \partial \phi_{n}}\right|_{\phi=\bar{\phi}}\left(\overline{\phi_{m} \phi_{n}}-\overline{\phi_{m} \phi_{n}}\right)=\left.\frac{1}{2} \frac{\partial^{2} p}{\partial \phi_{m} \partial \phi_{n}}\right|_{\phi=\bar{\phi}} \vartheta\left(\overline{\phi_{m}}, \overline{\phi_{n}}\right),
$$

where $m$ and $n$ index the components of vectors $\phi$ and $\psi$, and $\delta$ is the secondorder approximation in the LES assumption for the pressure. Extensive and tedious mathematics, the results of which appear in Appendix B, permits the computation of $\left(\partial^{2} p /\left(\partial \phi_{m} \partial \phi_{n}\right)\right)_{\phi=\bar{\phi}}$ from knowledge of $\left(\partial^{2} p /\left(\partial \psi_{m}^{\prime} \partial \psi_{n}^{\prime}\right)\right)_{\psi^{\prime}=\overline{\psi^{\prime}}}$ through the PR EOS. A one-dimensional test case for the PR EOS is presented in Appendix C, validating the utilization of the non-rigorous assumptions.

Figure 4 shows, as an example, the approximation of $\overline{p(\phi)}$ by $p(\bar{\phi})+\delta$ and compares it to that by $p(\bar{\phi})$ at two filter sizes $\bar{\Delta} / \Delta x=4,8$ for HN600 at $t_{t r}^{*}$. The qualitative conclusion from scrutiny of the plots is that the addition of $\delta$ is an improvement for the prediction of $\overline{p(\phi)}$ only at the small LES-filter size $\bar{\Delta} / \Delta x=4$ (DNS to LES computational grid volume increases by a factor of 64), since for $\bar{\Delta} / \Delta x=8$ (DNS to LES computational grid volume increases by a factor of 512) the term $\delta$ exceeds its physical value and generates unphysical oscillations. This conclusion is consistent with the one-dimensional analysis of Appendix C. To quantify the effect of the $\delta$ correction (equation (4.12)), listed in table 10 are the domain r.m.s. of the affected terms in the momentum and energy equations; all other terms' r.m.s. are unaffected by the $\delta$ model and their magnitude is listed in tables $5-8$.

For the momentum equation, an example comparison is that for the $x_{1}$ component by examining results for HN600 in tables 5 and 10. The terms with correction show that for $\bar{\Delta} / \Delta x=4$ when $\delta \neq 0, \nabla(\bar{p}-(p(\bar{\phi})+\delta))$ is only $36 \%$ of $\nabla(p(\bar{\phi})+\delta)$ and $16 \%$ of the leading-order term, although it is still larger than the subgrid flux which is though expected to increase at larger $R e$. By contrast, in the original equation $\nabla(\bar{p}-p(\bar{\phi}))$ is $57 \%$ of $\nabla(p(\bar{\phi})$ and $28 \%$ of the leading term, which means that for $\bar{\Delta} / \Delta x=4$ it is considerably more legitimate to neglect $\nabla(\bar{p}-(p(\bar{\phi})+\delta))$ in the $\delta$ modelled equation than it would be to neglect $\nabla(\bar{p}-p(\bar{\phi}))$ in the original equation. For $\bar{\Delta} / \Delta x=8$ and $i=1, \nabla(\bar{p}-(p(\bar{\phi})+\delta))$ is $86 \%$ of $\nabla(p(\bar{\phi})+\delta)$ and $76 \%$ of the leading term compared to the respective values of $78 \%$ and $52 \%$ in the original equation. Thus, at the larger filter size, the correction deteriorates the results, consistent with the visual information from figure 4.

For the energy equation, when $\delta \neq 0, \nabla \cdot((\bar{p}-(p(\bar{\phi})+\delta)) \widetilde{\boldsymbol{u}})$ is $5.4 \%$ and $23 \%$ of $\nabla \cdot((p(\bar{\phi})+\delta) \widetilde{\boldsymbol{u}})$ for $\bar{\Delta} / \Delta x=4$ and 8 , respectively, whereas in the original LES equation the corresponding values are $11 \%$ and $19.6 \%$; however, in all cases, these 

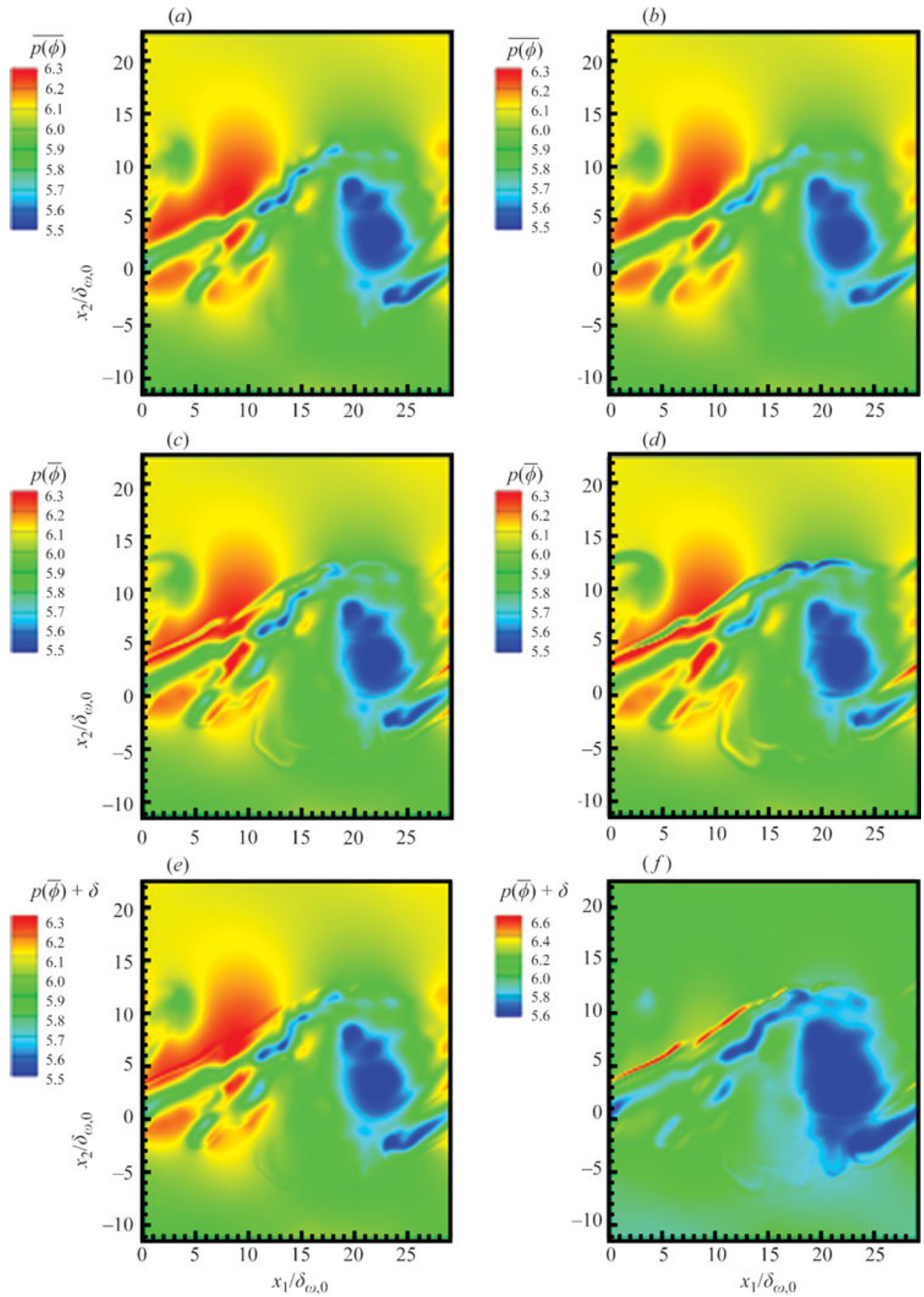

FIGURE 4. Approximation of $\overline{p(\phi)}(a, b)$ by $p(\bar{\phi})(c, d)$ and $p(\bar{\phi})+\delta(e, f)$ at two filter sizes: $\bar{\Delta}=4 \Delta x(a, c, e)$ and $\bar{\Delta}=8 \Delta x(b, d, f)$ for the simulation HN600 at $t_{t r}^{*}$. Units are MPa.

terms are at least one order of magnitude smaller than the leading, convective term (see table 8). This correction does not seem necessary in the original LES energy equation but must be included with that of the momentum equation, for consistency. 


\begin{tabular}{|c|c|c|c|c|}
\hline & & $\bar{\Delta}=4 \Delta x$ & $\bar{\Delta}=8 \Delta x$ & equation are $g$ \\
\hline$x_{1}$-momentum & $\frac{\partial}{\partial x_{1}}(p(\bar{\phi})+\delta)$ & 10.8 & 17.7 & table 5 \\
\hline$x_{1}$-momentum & $\frac{\partial}{\partial x_{1}}(\bar{p}-(p(\bar{\phi})+\delta))$ & 3.92 & 15.3 & table 5 \\
\hline$x_{2}$-momentum & $\frac{\partial}{\partial x_{2}}(p(\bar{\phi})+\delta)$ & 12.8 & 23.3 & table 6 \\
\hline$x_{2}$-momentum & $\frac{\partial}{\partial x_{2}}(\bar{p}-(p(\bar{\phi})+\delta))$ & 5.55 & 21.0 & table 6 \\
\hline$x_{3}$-momentum & $\frac{\partial}{\partial x_{3}}(p(\bar{\phi})+\delta)$ & 11.4 & 19.5 & table 7 \\
\hline$x_{3}$-momentum & $\frac{\partial}{\partial x_{3}}(\bar{p}-(p(\bar{\phi})+\delta))$ & 5.20 & 17.8 & table 7 \\
\hline energy & $\frac{\partial}{\partial x_{j}}\left((p(\bar{\phi})+\delta) \tilde{u_{j}}\right)$ & 4.01 & 4.14 & table 8 \\
\hline energy & $\frac{\partial}{\partial x_{j}}\left[(\bar{p}-(p(\bar{\phi})+\delta)) \tilde{u}_{j}\right]$ & 0.216 & 0.953 & table 8 \\
\hline
\end{tabular}

TABLE 10. Magnitude (r.m.s.) of terms in the momentum and energy equations for HN600 with the correction from the Taylor expansion (i.e. $\delta$ is non-null). The r.m.s. is presented for the two LES-filter widths. See tables 5-8 for the HN600 r.m.s. of all other terms in the corresponding equations, as they are independent of the $\delta$ model. Units are $10^{6} \mathrm{~N} \mathrm{~m}^{-3}$ for the momentum equation and $10^{9} \mathrm{~J} \mathrm{~m}^{-3} \mathrm{~s}^{-1}$ for the energy equation.

The conclusions are then that the energy equation is much less affected by the correction than the momentum equation, and that at larger filters this correction is not advisable, at least for the momentum equation.

The attractiveness of the equation (4.12) model is the availability of variances $\vartheta$ from the SGS modelling approach (see §4.2.1). Higher-order corrections in the Taylor expansion of equation (4.10) may also improve the approximation, but would remove the benefit of computational efficiency because higher-order variances would be required. Thus, the balance between computational accuracy and efficiency means that with a small computational effort we might capture the essence of the physics; the extent to which this physics is captured will require further a posteriori study evaluations.

Generally, when conducting LES one does not have the present guiding information of a similar DNS, and it is thus impossible to determine prior to the computation what filter size may be excessive in a supercritical flow calculation. Therefore, it is foreseen that for supercritical-flow LES, grid resolution studies will be even more crucial than at atmospheric pressure to ensure that the computation is well resolved at the LES scale.

\subsubsection{Modelling of $\nabla \cdot\left[\boldsymbol{q}_{I K}-\boldsymbol{q}_{I K}(\bar{\phi})\right]$}

When examining the heat flux term in equations $(2.11)-(2.14)$, the difference in complexity between modelling $\nabla[\bar{p}-p(\bar{\phi})]$ and modelling $\nabla \cdot\left[\boldsymbol{q}_{I K}-\boldsymbol{q}_{I K}(\bar{\phi})\right]$ becomes quickly apparent. That is, whereas according to equation $(3.1) p(\psi)$ is a function of the thermodynamic primitive variables, $\boldsymbol{q}_{I K}(\psi)$ is a function of both the thermodynamic primitive variables and thermodynamic primitive variable gradients. Under the high$p$ and moderate $\rho_{r} \equiv \rho / \rho_{c}$ (i.e. $O\left(10^{-1}\right)$ ) conditions of this study, the transport 
coefficients $\alpha_{I K}$ or $\alpha_{B K}$ appearing in equations (2.12)-(2.14) cannot generally be expressed as accepted and/or comprehensive functions of thermodynamic variables in $\psi$ (e.g. Gonzales-Bagnoli, Shapiro \& Stenby 2003). In the present DNS, $\alpha_{I K}$ or $\alpha_{B K}$ were specified as constants and, for computational efficiency, the transport coefficients $\lambda$ and $D$ were computed from the correlations of table 3 , meaning that the complex dependence of all transport coefficients on thermodynamic variables in $\psi$ has been simplified by a curve fit which makes some explicit dependences unavailable. Faced with this modelling challenge, several approaches were assessed, as described below.

In a first approach, the approximation

$$
\overline{\boldsymbol{q}_{I K}(\psi)} \simeq \overline{C_{T}(\psi)} \nabla T(\bar{\phi})+\overline{C_{P}(\psi)} \nabla p(\bar{\phi})+\overline{C_{Y}(\psi)} \nabla Y_{2}(\bar{\phi})
$$

was considered with each function $\overline{C_{T}(\psi)}, \overline{C_{P}(\psi)}$ and $\overline{C_{Y}(\psi)}$ modelled following a Taylor series similar to equation (4.10), leading to $\delta_{T}, \delta_{P}$ and $\delta_{Y}$; for example,

$$
\left.\delta_{T} \equiv \frac{1}{2} \frac{\partial^{2} C_{T}}{\partial \phi_{m} \partial \phi_{n}}\right|_{\psi=\bar{\psi}}\left(\overline{\phi_{m} \phi_{n}}-\overline{\phi_{m} \phi_{n}}\right)=\left.\frac{1}{2} \frac{\partial^{2} C_{T}}{\partial \phi_{m} \partial \phi_{n}}\right|_{\psi=\bar{\psi}} \vartheta\left(\overline{\phi_{m}}, \overline{\phi_{n}}\right) .
$$

Since owing to the aforementioned lack of knowledge regarding the transport coefficients dependence on $\psi$, the analytical functions $C_{T}(\phi), C_{P}(\phi)$ and $C_{Y}(\phi)$ are typically not available, a numerical multivariate differentiation technique was used to obtain the results. When the DNS-computed $\left(\overline{C_{T}(\phi)}-C_{T}(\bar{\phi})\right),\left(\overline{C_{P}(\phi)}-C_{P}(\bar{\phi})\right)$ and $\left(\overline{C_{Y}(\phi)}-C_{Y}(\bar{\phi})\right)$ were compared in $\left(x_{1}, x_{2}\right)$-planes to the corresponding modelled $\delta_{T}, \delta_{P}$ and $\delta_{Y}$, the results, shown in figure 5 for $\mathrm{OH} 500$, were very encouraging, definitely validating the Taylor series methodology as well as the numerical differentiation technique. The upper free-stream discrepancy between the modelled $\delta_{Y}$ and its exact value is not of great concern since $\nabla Y_{2}$ is null in those regions. Unfortunately, when these results were used in conjunction with a Taylor expansion of the entire heat flux according to equation (4.13), the DNS-extracted difference $\left(\overline{q_{I K i}(\phi)}-q_{I K i}(\bar{\phi})\right)$ did not compare well with $\delta_{i}$ (not shown), indicating that this approach may not be appropriate.

The second approach involved the Taylor expansion differentiation applied to the entire heat flux, for each component, but the model again did not duplicate the DNS-extracted result (not shown).

A third approach was based on re-thinking the entire modelling methodology when both primitive variables and their gradients are involved. Thus, a Taylor series approach was devised where $\phi$ and $\nabla \phi$ are treated as independent variables. The heat-flux vector can be formally written as

$$
q_{I K i}(\phi)=\sum_{q} C_{q}(\phi) \frac{\partial F_{q}(\phi)}{\partial x_{i}}
$$

where $q$ is an index and $C_{1}(\phi)=C_{T}(\phi), C_{2}(\phi)=C_{P}(\phi), C_{3}(\phi)=C_{Y}(\phi), F_{1}(\phi)=T(\phi)$, $F_{2}(\phi)=p(\phi)$ and $F_{3}(\phi)=Y_{2}$. Using the notation

$$
\delta F_{q}=F_{q}(\phi)-F_{q}(\bar{\phi})
$$

the heat flux is

$$
\begin{aligned}
q_{I K i}(\phi) & =\sum_{q} C_{q}(\phi) \frac{\partial F_{q}(\bar{\phi})}{\partial x_{i}}+\sum_{q} C_{q}(\phi) \frac{\partial \delta F_{q}}{\partial x_{i}} \\
& =\sum_{q} C_{q}(\phi) \frac{\partial F_{q}(\bar{\phi})}{\partial x_{i}}+\frac{\partial}{\partial x_{i}} \sum_{q} C_{q}(\phi) \delta F_{q}-\sum_{q} \delta F_{q} \frac{\partial C_{q}(\phi)}{\partial x_{i}}
\end{aligned}
$$


(a)
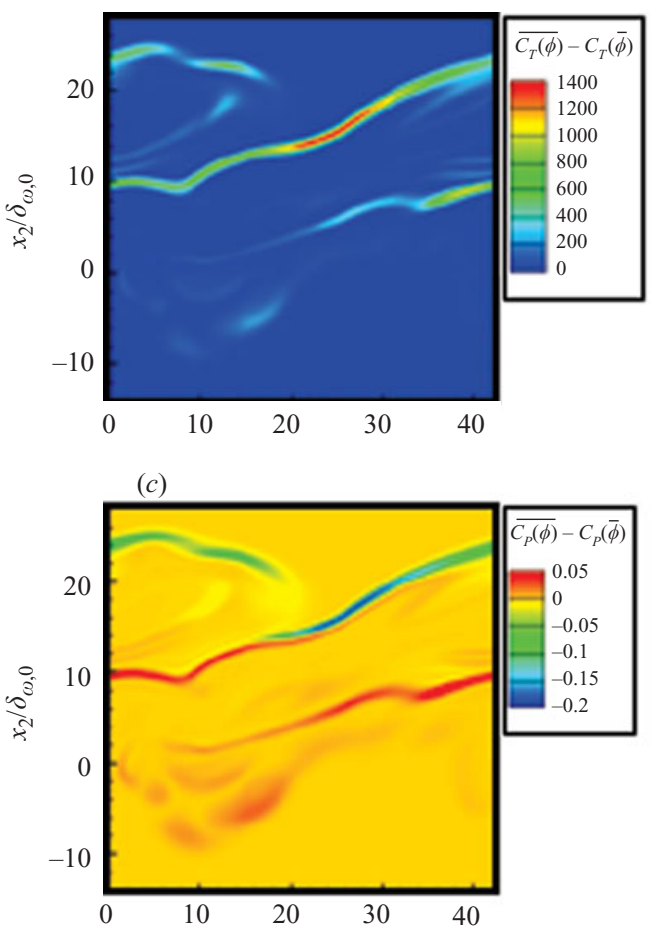

(e)

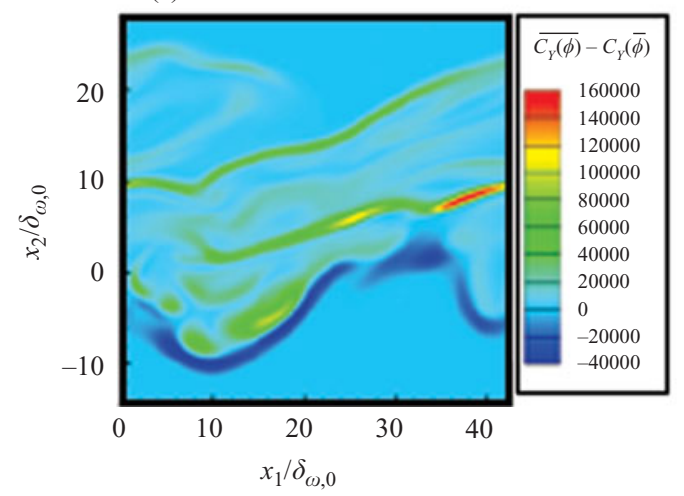

(b)
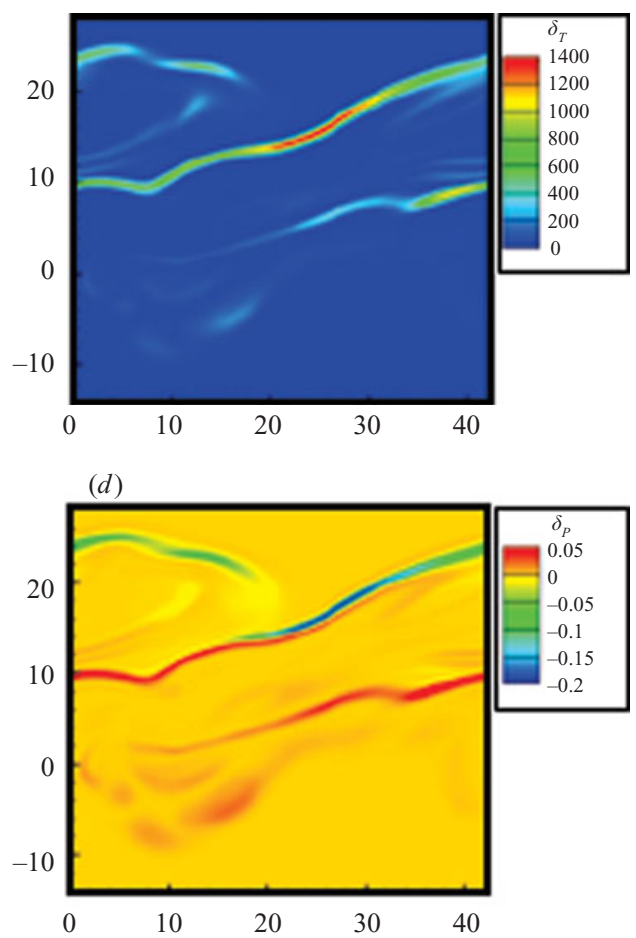

( f)

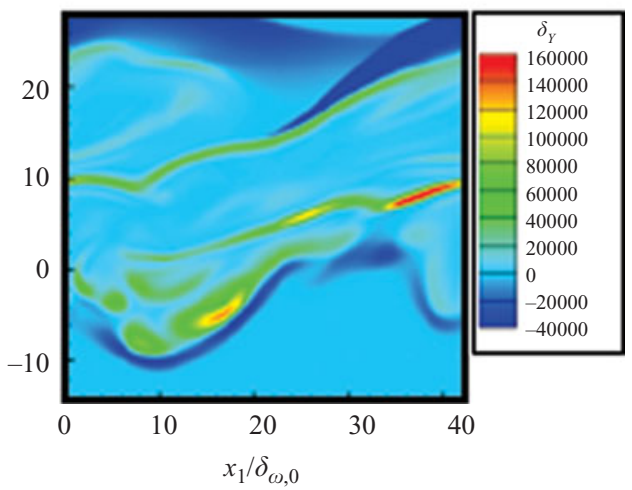

Figure 5. Taylor expansion of the heat-flux coefficients $C_{T}, C_{P}$ and $C_{Y}$ from equation (2.11) calculated for $\mathrm{OH} 500$ using numerical differentiation: $(a, c, e)$ are the DNS-extracted values and $(b, d, f)$ are calculated using the model of equation (4.14) for $\delta_{T}$ and similar equations for $\delta_{P}$ and $\delta_{Y}$.

and through filtering one obtains

$$
\overline{q_{I K i}(\phi)}=\underbrace{\sum_{q} \overline{C_{q}(\phi)} \frac{\partial F_{q}(\bar{\phi})}{\partial x_{i}}}_{\text {Part } 1}+\underbrace{\frac{\partial}{\partial x_{i}} \sum_{q} \overline{C_{q}(\phi) \delta F_{q}}}_{\text {Part } 2}-\underbrace{\sum_{q} \overline{\delta F_{q} \frac{\partial C_{q}(\phi)}{\partial x_{i}}}}_{\text {Part } 3} .
$$

Based on an a priori evaluation of the DNS-extracted values showing that for all $\mathrm{OH}$ simulations the term $\sum_{q} \overline{C_{q}(\phi) \nabla F_{q}(\bar{\phi})}$ always contains the bulk of the heat flux, the split of equation (4.19) for $q_{i}$ seemed promising because the indication is that 
Part 1 in equation (4.19) may also contain the bulk of $q_{i}$. Then, Part 1 could be modelled using the Taylor expansion of $C_{q}(\phi)$, which has been shown successful (figure 5). Rigorous computation of Parts 2 and 3 of equation (4.19) through a Taylor expansion is not possible because each Part is a sum of numerous terms, only one of which is calculable; the other terms involve higher-order correlations of $\phi$ and $F(\phi)$, which are unknown. One pragmatic way of modelling the heat flux would be to include only those terms for which a closed form is available and neglect the other terms. However, based on the present a priori analysis, given the computational cost of the numerical differentiation (where a second-order function differentiation involves the computation of that function at 12 locations) and the change of variables between the thermodynamic vector $\psi$ and the conservative vector $\phi$, the preliminary evaluation is that such heat flux models may be prohibitively expensive in a LES; further a posteriori studies are needed for a more definitive answer.

Equations (4.6)-(4.9) still contain unclosed terms, namely the SGS fluxes and variances in equations (4.6)-(4.9) and the variances needed to calculate $\delta$ in equation (4.12), all of which will be modelled explicitly.

\subsection{Subgrid flux and variance modelling}

\subsubsection{SGS-flux models}

The three basic models for the SGS fluxes $\left(\tau_{i j}, \eta_{\alpha j}, \zeta_{j}\right)$ are (Okong'o \& Bellan 2004b) the Smagorinsky (SM) model, the Gradient (GR) model and the Scale-Similarity (SS) model. For the a priori analysis, only constant-coefficient versions of these models can be considered, although dynamic-coefficient versions should also be studied in a posteriori LES (Leboissetier, Okong'o \& Bellan 2005). Because dynamic models are based on the same concept as the SS model, the a priori evaluation of that model should provide reasonable indications of the likely performance of dynamic models. Although here the constant coefficients are calibrated, other effects that cannot be studied a priori, such as the interaction of the resolved flow with the SGS, may dictate a different value in actual LES. The calibration will consider the same coefficient value for all SGS fluxes, although practical implementation may require different values for different fluxes, in addition to the spatial and temporal variation of coefficients that is afforded by dynamic modelling (Leboissetier et al. 2005).

The SM model is based on the gradient-diffusion (eddy-viscosity) concept (Smagorinksy 1993). As such, it does not lend itself to computing variances in general. The SGS fluxes in equation (2.26) are

$$
\vartheta_{S M}\left(\widetilde{\psi}_{m}, \tilde{u}_{j}\right)=-C_{S M} \bar{\Delta}^{2} S(\bar{\phi}) \frac{1}{2} \frac{\partial \widetilde{\psi}_{m}}{\partial x_{j}}, \psi_{m} \neq u_{j},
$$

with $\tau_{i j}$ modelled in trace-free form as

$$
\tau_{i j}-\frac{1}{3} \tau_{k k} \delta_{i j}=-C_{S M} \bar{\Delta}^{2} S(\bar{\phi})\left[S_{i j}(\bar{\phi})-\frac{1}{3} S_{k k}(\bar{\phi}) \delta_{i j}\right],
$$

where $S^{2}(\phi)=S_{i j}(\phi) S_{i j}(\phi)$. The Yoshizawa (1986) (YO) model for $\tau_{k k}$ is

$$
\tau_{k k}=C_{Y O} \bar{\Delta}^{2} S^{2}(\bar{\phi}) \text {. }
$$

The GR model, derived from a Taylor series expansion, is (Clark, Ferziger \& Reynolds (1979)

$$
\vartheta_{G R}\left(\widetilde{\psi}_{m}, \widetilde{\psi}_{n}\right)=C_{G R} \bar{\Delta}^{2} \frac{\partial \tilde{\psi}_{m}}{\partial x_{k}} \frac{\partial \tilde{\psi}_{n}}{\partial x_{k}} .
$$

(Note: $\vartheta\left(\tilde{u}_{1}, \tilde{u}_{1}\right)=\tau_{11}, \vartheta\left(\tilde{u}_{2}, \tilde{u}_{2}\right)=\tau_{22}, \vartheta\left(\tilde{u}_{3}, \tilde{u}_{3}\right)=\tau_{33}$.) Theoretically, $C_{G R}$ is proportional to the moments of inertia of the filtering volume; for a cubic top-hat filter $C_{G R}=1 / 12$ (Okong'o \& Bellan 2004b). 


$\begin{array}{lcccc}\text { SGS quantity } & \text { SM } & \text { GR } & \text { SS }(\hat{\Delta}=\bar{\Delta}) & \text { SS }(\hat{\Delta}=2 \bar{\Delta}) \\ \bar{\rho} \tau_{11} & 0.0633 & 0.1269 & 1.4741 & 0.5141 \\ \bar{\rho} \tau_{22} & 0.0241 & 0.1241 & 1.5030 & 0.5667 \\ \bar{\rho} \tau_{33} & 0.0160 & 0.1210 & 1.4826 & 0.5574 \\ \bar{\rho} \tau_{12} & 0.0366 & 0.1133 & 1.3331 & 0.4572 \\ \bar{\rho} \tau_{13} & 0.0241 & 0.1158 & 1.4547 & 0.5348 \\ \bar{\rho} \tau_{23} & 0.0160 & 0.1143 & 1.5005 & 0.6108 \\ \bar{\rho} \zeta_{1} & 0.1662 & 0.1200 & 1.4647 & 0.5132 \\ \bar{\rho} \zeta_{2} & 0.0607 & 0.1143 & 1.4389 & 0.4802 \\ \bar{\rho} \zeta_{3} & 0.0522 & 0.1119 & 1.4371 & 0.4812 \\ \bar{\rho} \eta_{1} & 0.1683 & 0.1201 & 1.4641 & 0.5132 \\ \bar{\rho} \eta_{2} & 0.0591 & 0.1145 & 1.4378 & 0.4805 \\ \bar{\rho} \eta_{3} & 0.0509 & 0.1122 & 1.4360 & 0.4810 \\ \vartheta\left(\tilde{u}_{1}, \tilde{u}_{1}\right) & - & 0.1200 & 1.4174 & 0.5088 \\ \vartheta\left(\tilde{u}_{2}, \tilde{u}_{2}\right) & - & 0.1213 & 1.4535 & 0.5516 \\ \vartheta\left(\tilde{u}_{3}, \tilde{u}_{3}\right) & - & 0.1190 & 1.4596 & 0.5602 \\ \vartheta(\tilde{T}, \tilde{T}) & - & 0.1204 & 1.7133 & 0.7200 \\ \vartheta\left(\tilde{Y}, \tilde{Y}_{2}\right) & - & 0.1136 & 1.5360 & 0.6143 \\ \vartheta(\bar{p}, \bar{p}) & - & 0.1224 & 1.4021 & 0.5197 \\ \text { Average slope } & 0.0622 & 0.1180 & 1.4671 & 0.5369 \\ \text { Std. dev. of slopes } & 0.0577 & 0.0044 & 0.0230 & 0.0636 \\ \text { Average correlation } & 0.2313 & 0.9602 & 0.9492 & 0.8322\end{array}$

TABLE 11. Slopes from least-squares fit of SGS models to SGS quantities (slope = exact $/ \mathrm{model}$ ), OHe600, $\bar{\Delta} / \Delta x=8$. For simplification, the vector $\eta_{2}$ is simply denoted as $\eta$. The SM model $\tau_{i j}$ is compared to the exact $\left(\tau_{i j}-\tau_{k k} \delta_{i j} / 3\right)$. For $\bar{\rho} \tau_{k k}$ using the YO model, the slope is 0.2275 and the correlation is 0.8332 .

The SS model, which postulates similarity between the SGS and the small resolved scale, is (Bardina, Ferziger \& Reynolds 1980)

$$
\vartheta_{S S}\left(\widetilde{\psi}_{m}, \widetilde{\psi}_{n}\right)=C_{S S}\left(\widetilde{\psi}_{m} \tilde{\psi}_{n}-\widehat{\tilde{\psi}}_{m} \widehat{\tilde{\psi}}_{n}\right),
$$

where the hat denotes (unweighted) filtering at the test-filter level $\hat{\Delta}$. Two test-filter widths are considered, leading to models $\operatorname{SS} 1(\hat{\Delta} / \bar{\Delta}=1)$ and $\operatorname{SS} 2(\hat{\Delta} / \bar{\Delta}=2)$. While scale-similarity would imply that $C_{S S}=1$, the actual value is filter-width dependent (Liu, Meneveau \& Katz 1994; Pruett, Sochacki \& Adams 2001; Okong'o \& Bellan 2004b).

Least-squares fits of the exact SGS fluxes to the SGS-flux models produced the slope (exact/model) and correlation for each SGS quantity; the model coefficient is the slope from the least-squares fit. For each SGS model, the calibrated SGS coefficient for a given run and filter width is obtained by averaging the slopes obtained for each SGS quantity. The SM coefficient is based on $12 \mathrm{SGS}$ quantities (six independent $\tau_{i j}$, three $\zeta_{j}$, three $\eta_{\alpha j}$ ), whereas the GR and SS coefficients are based on an additional six SGS variances. Owing to the strong density variation, the actual calibration is performed for the product of density and SGS flux, that appears in equations (4.7)(4.9). The slopes and the average of the correlations are listed for OHe600 (the DNS having the largest $R e_{m, t r}$ ) in table 11. The correlations for the SS and GR models are typically better than $95 \%$ (better than $80 \%$ for SS2), whereas the correlations for the SM model are at best $50 \%$ and are typically about $20 \%$. Whereas the GR and SS slopes have a narrow distribution, as indicated by their small standard deviation of the slopes, there is wide variation among SM slopes, with the standard deviation of 

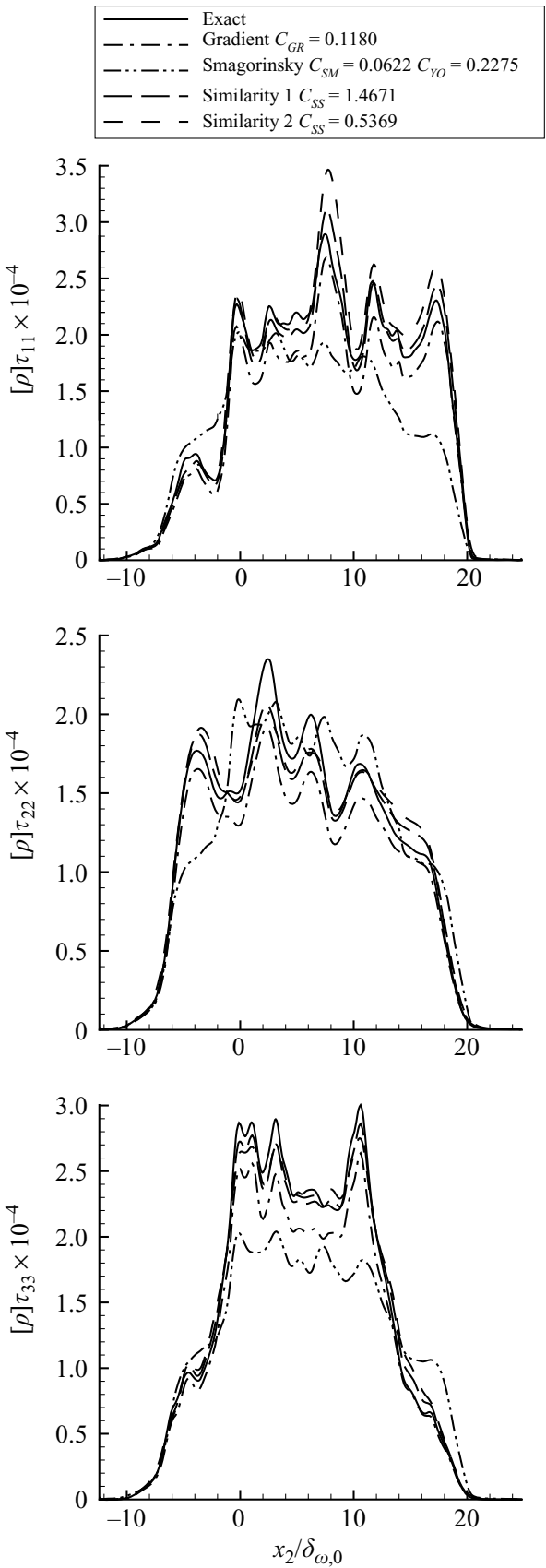
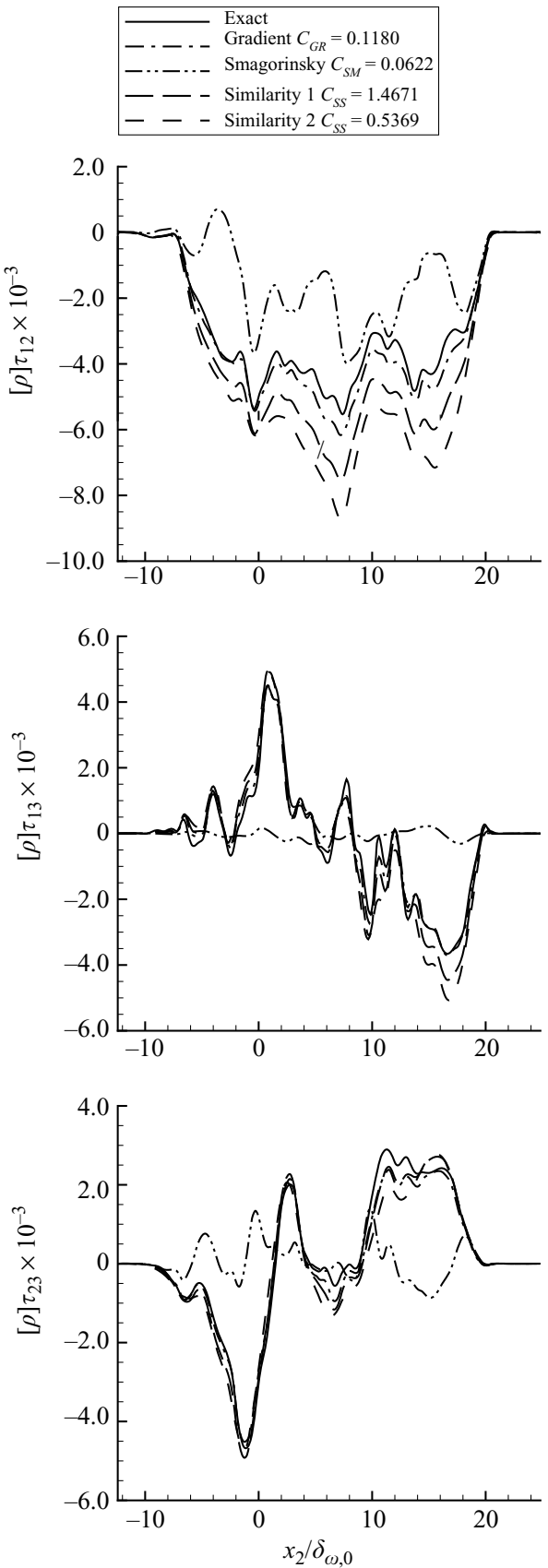

FIGURE 6. SGS fluxes and models for OHe600 with $\bar{\Delta}=8 \Delta x$, averages in homogeneous planes at $t_{t r}^{*}$. The notation $[\rho] \equiv \bar{\rho}$ is used. Units are $\mathrm{Nm}^{-2}$.

the SM slopes being comparable in magnitude to the average. These characteristics of OHe600 are typical of all layers at both filter widths.

Figures 6 and 7 compare the different SGS-flux models in terms of their averages in homogeneous $\left(x_{1}, x_{3}\right)$-planes for OHe600. The calibrated coefficient values (that is, the average slopes) from table 11 are used for the comparison. The Smagorinsky model has poor agreement with the exact (computed) SGS fluxes for all components, consistent 

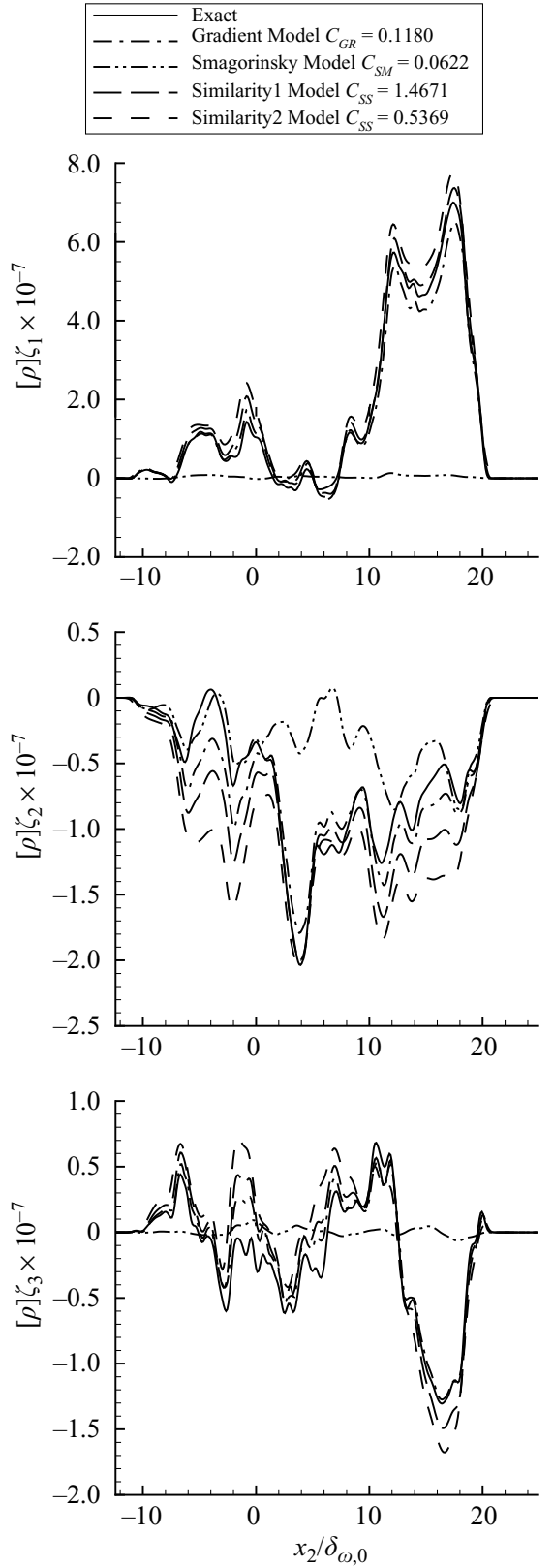
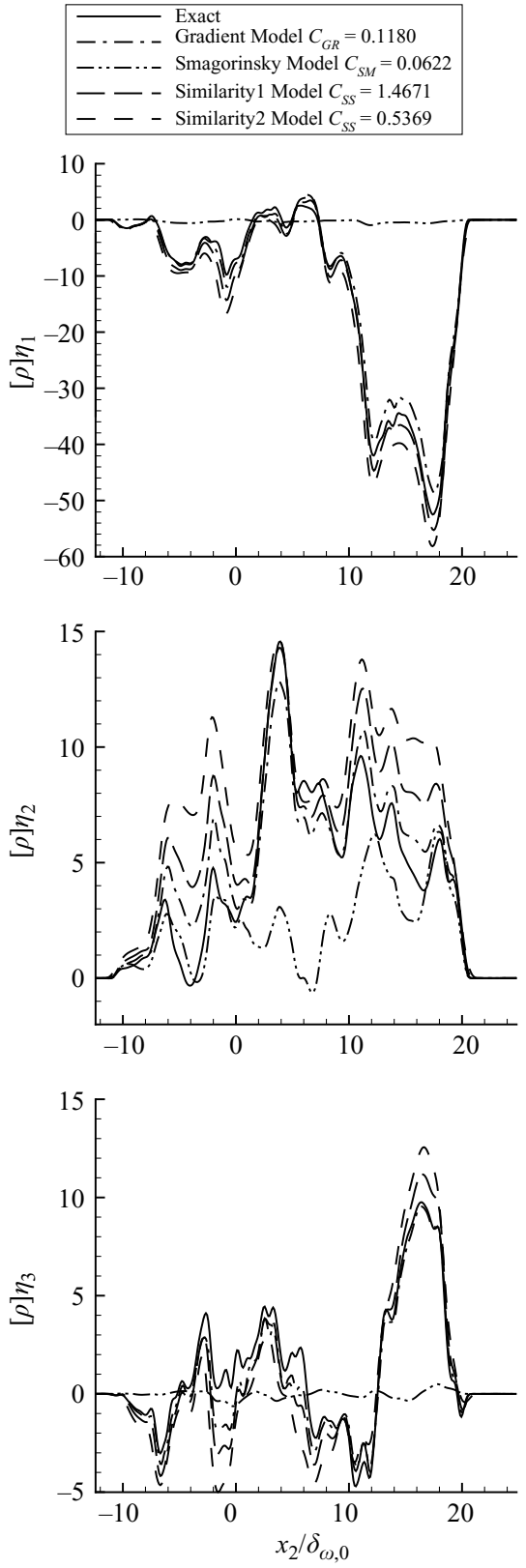

FIGURE 7. SGS fluxes and models for OHe600 with $\bar{\Delta}=8 \Delta x$, averages in homogeneous planes at $t_{t r}^{*}$. For simplification, the vector $\eta_{2}$ is simply denoted as $\eta$ and the notation $[\rho] \equiv \bar{\rho}$ is used. Units are $\left(\mathrm{J} \mathrm{m}^{-2} \mathrm{~s}^{-1}\right)$ for $[\rho] \zeta$ and $\left(\mathrm{kg} \mathrm{m}^{-2} \mathrm{~s}^{-1}\right)$ for $[\rho] \eta$.

with the low correlations; its deficiencies cannot be remedied by simply using different coefficient values for the different types of fluxes. However, the Yoshizawa model correlates quite well (over $80 \%$ ) with $\tau_{k k}$, and in this case, where $\tau_{k k}$ dominates in $\tau_{i j}$, the combination with the Smagorinsky model yields good predictions of $\tau_{11}, \tau_{22}$, and $\tau_{33}$. In marked contrast to the Smagorinsky model, the SS and GR models clearly have both qualitatively and quantitatively good agreement with the exact SGS fluxes for all components. 


$\begin{array}{lcccccccc}\text { Run } & \text { HN400 } & \text { HN500 } & \text { HN600 } & \text { HN800 } & \text { OH750 } & \text { OH550 } & \text { OH500 } & \text { OHe600 } \\ C_{Y O}(\bar{\Delta} / \Delta x=4) & 0.2751 & 0.2687 & 0.2583 & 0.2612 & 0.2396 & 0.2383 & 0.2398 & 0.2477 \\ C_{Y O}(\bar{\Delta} / \Delta x=8) & 0.2751 & 0.2634 & 0.2471 & 0.2506 & 0.2144 & 0.2150 & 0.2137 & 0.2275 \\ C_{S M}(\bar{\Delta} / \Delta x=4) & 0.0726 & 0.0735 & 0.0655 & 0.0409 & 0.1442 & 0.1315 & 0.1293 & 0.0711 \\ C_{S M}(\bar{\Delta} / \Delta x=8) & 0.0742 & 0.0687 & 0.0579 & 0.0423 & 0.1232 & 0.1169 & 0.1138 & 0.0622 \\ C_{G R}(\bar{\Delta} / \Delta x=4) & 0.1372 & 0.1397 & 0.1346 & 0.1344 & 0.1284 & 0.1280 & 0.1275 & 0.1328 \\ C_{G R}(\bar{\Delta} / \Delta x=8) & 0.1254 & 0.1257 & 0.1193 & 0.1180 & 0.1115 & 0.1118 & 0.1112 & 0.1180 \\ C_{S S}(\hat{\Delta} / \bar{\Delta}=1, \bar{\Delta} / \Delta x=4) & 1.3352 & 1.3293 & 1.2938 & 1.2954 & 1.1312 & 1.1048 & 1.0983 & 1.2388 \\ C_{S S}(\hat{\Delta} / \bar{\Delta}=1, \bar{\Delta} / \Delta x=8) & 1.6891 & 1.6069 & 1.5399 & 1.5661 & 1.2920 & 1.2560 & 1.2448 & 1.4671 \\ C_{S S}(\hat{\Delta} / \bar{\Delta}=2, \bar{\Delta} / \Delta x=4) & 0.4934 & 0.4904 & 0.4705 & 0.4676 & 0.3845 & 0.3685 & 0.3655 & 0.4426 \\ C_{S S}(\hat{\Delta} / \bar{\Delta}=2, \bar{\Delta} / \Delta x=8) & 0.6998 & 0.6260 & 0.5770 & 0.5870 & 0.4469 & 0.4303 & 0.4243 & 0.5369\end{array}$

TABLE 12. Model cofficients calibrated from transitional states.

The calibrated coefficients for all layers (see table 2) are tabulated in table 12. The calibrated coefficients are here compared to determine possible statistical equality of the values (based on t-tests with $5 \%$ confidence level). The YO coefficients range from 0.2137 to 0.2751 , with the lower values for $\mathrm{OH}$ at the larger $\bar{\Delta} / \Delta x$, while the $\mathrm{HN}$ values show little filter-width dependence. At fixed $\bar{\Delta} / \Delta x$, the OHe600 coefficient lies between the $\mathrm{HN}$ and $\mathrm{OH}$ values, and is approximately equal to the average coefficient computed over all layers; this behaviour was also observed for all the other models. Except for the SM model, where the trend is reversed, for a given $\bar{\Delta} / \Delta x$, the HN values are higher than the $\mathrm{OH}$ values. The range of coefficient values is $0.0409-0.1442$ (SM), 0.1112-0.1397 (GR), 1.0988-1.6891 (SS1) and 0.3655-0.6998 (SS2). For the SM model, the coefficients are statistically independent of run and filter width, because the underlying SM coefficients have a large spread of slopes (large standard deviation, e.g. table 11 for OHe600). This result indicates that the correlation of the SM model with the SGS fluxes is too poor for this calibration procedure to produce a meaningful coefficient.

For the GR and SS models, the statistical equivalence of the coefficients in table 12 mirrors the closeness of numerical values, due to the small variation (small standard deviation) in the underlying slopes. For both models, the coefficients are filter-width dependent for each run. At either $\bar{\Delta} / \Delta x$, the three $\mathrm{OH}$ coefficients are (statistically) equal, the $\mathrm{HN}$ coefficients are also generally equal, and those of $\mathrm{OHe} 600$ are generally equal to the closest $\mathrm{HN}$ value (HN600 or HN800). For the GR model, the HN400 and HN500 values at $\bar{\Delta} / \Delta x=8$ are equal to the $\mathrm{OH}$ values at $\bar{\Delta} / \Delta x=4$. For the SS1 model, the HN values at $\bar{\Delta} / \Delta x=4$ are equal to the OH750 value at $\bar{\Delta} / \Delta x=8$. For the SS2 model, the OHe600 value at $\bar{\Delta} / \Delta x=4$ is equal to the $\mathrm{OH}$ values at $\bar{\Delta} / \Delta x=8$. Based on the $\bar{\Delta} / \Delta x$ - and run-dependence of the GR coefficients, it is anticipated that dynamic modelling, wherein the model coefficient is computed during LES from the LES flow field, will be required. Because dynamic modelling is based on the SS model with $C_{S S}=1$, the fact that the SS1 coefficient values are closer to unity than are the SS2 values suggests that $\hat{\Delta}=\bar{\Delta}$ has the greater potential for dynamic modelling. An a posteriori study is needed to determine the sensitivity of the LES to the model coefficients.

\subsubsection{Variance modelling for $\delta$}

Variances appearing in equation (4.12) are $\vartheta(\bar{\rho}, \bar{\rho}), \quad \vartheta\left(\overline{\rho Y_{2}}, \overline{\rho Y_{2}}\right), \quad \vartheta(\overline{\rho e}, \overline{\rho e})$, $\vartheta\left(\bar{\rho}, \overline{\rho Y_{2}}\right), \vartheta(\bar{\rho}, \overline{\rho e})$ and $\vartheta\left(\overline{\rho Y_{2}}, \overline{\rho e}\right)$. Because the goal of LES is to minimize the 


\begin{tabular}{lcccccccccc} 
& \multicolumn{4}{c}{$\bar{\Delta}=4 \Delta x$} & & \multicolumn{5}{c}{$\bar{\Delta}=8 \Delta x$} \\
\cline { 2 - 7 } \cline { 8 - 10 } Runs & HN400 & HN500 & HN600 & HN800 & & HN400 & HN500 & HN600 & HN800 \\
mean & 0.1491 & 0.1514 & 0.1493 & 0.1505 & & 0.1460 & 0.1463 & 0.1444 & 0.1423 \\
stand. dev. & 0.0056 & 0.0048 & 0.0036 & 0.0059 & & 0.0065 & 0.0078 & 0.0053 & 0.0104
\end{tabular}

TABLE 13. Means and standard deviations of slopes (exact/model) for the variances used in the calculation of the modelled pressure gradient difference term in the momentum, equation (4.14), utilizing the GR model, equation (4.25).

computational time, and since enthalpy-based variances are already calculated as part of SGS-flux modelling, an assessment was made of whether $e$-based variances can be replaced by $h$-based ones. Results obtained at two filter sizes (not shown) reveal that the terms representing the difference between $e$ - and $h$-based variances are at least 3 orders of magnitude smaller than the variances, and thus negligible for both filter sizes. The explanation for this good approximation lies in the fact that the length scale of the pressure fluctuations is much larger than the LES filter, which leads to negligible $\vartheta(\bar{\varphi}, \bar{p})$ for $\varphi=\rho$ or $\rho Y_{2}$.

The GR model that best modelled the SGS fluxes in $\S 4.2 .1$ was used here to assess its ability to model the variances needed to calculate $\delta$. The entire ensemble of variances was used, and the correlation and calibrating coefficient were calculated through a least-square fit, the slope (exact/model) being the calibrated coefficient. The correlations' range is 0.983 to 0.994 for $\bar{\Delta} / \Delta x=4$ and 0.949 to 0.977 for $\bar{\Delta} / \Delta x=8$, and is thus considered excellent. A summary of the mean and standard deviation of the slopes is listed in table 13 for HN layers. The value of the slopes' standard deviation being much smaller than the mean, all mean values are statistically significant. Double-tail t-tests show that for any specific case the coefficient mean is statistically significant across filter sizes. For a given filter size, the coefficients are not statistically significant across the entire set of $\mathrm{HN}$ simulations because of the very small standard deviation for each case, whereas they are statistically significant across $\mathrm{OH}$ simulations; at similar $R e_{m, t r}$ (HN600 versus OH750), there is calibrating-coefficient statistical significance. Furthermore, comparing to the SGS-flux values listed for the GR model in table 12, the present coefficients are somewhat larger, indicating that it is unlikely that a constant-coefficient approach for the global model will be feasible. Moreover, the calibrated coefficients represent values found at transitional states, and forewarning exists from a posteriori studies at atmospheric conditions (Leboissetier et al. 2005) that model coefficients display substantial temporal variation, even for a specific variance, over the flow development time. Nevertheless, the question of whether dynamic SGS modelling is required should be addressed in future a posteriori studies of supercritical mixing layers.

\subsection{Scalar dissipation}

Small-scale mixing, which must be reproduced in LES by the SGS models, is directly associated with the scalar dissipation. The questions we ask are: Does the scalar dissipation distribution in supercritical turbulent flows have the well-known filamentary aspect of atmospheric flows (e.g. Su \& Clemens 2003)? And if so, is the scalar distribution probability density function (PDF) akin to the Gaussian that it resembles in turbulent atmospheric-pressure flows, except for the small non-null skewness (Warhaft 2000; Su \& Clemens 2003)? Although a Gaussian is expected 
only in homogeneous isotropic flows, the nearly Gaussian form observed in nonhomogeneous turbulent atmospheric-pressure flows allowed the utilization of this convenient mathematical form for flamelet modelling (Peters 2000), although Selle \& Bellan (2007) showed that the $\beta$-density PDF in a modified system of variables is a much better fit for the exact atmospheric-pressure PDF for both single-phase and two-phase flows. If the answer to both questions above is positive, then perhaps the specific supercritical SGS modelling departure from atmospheric-pressure flows does not have much impact on accurate small-scale mixing representation in LES. The present DNS database is amenable to analysis for answering these questions.

The scalar dissipation is proportional to the dissipation associated with the species fluxes and is defined as (Okong'o \& Bellan 2002a)

$$
\chi \equiv \frac{1}{\rho D} j_{\alpha j} j_{\alpha j} .
$$

Instead of the single term representing $\chi$ under atmospheric conditions, now $\chi$ is the sum of six terms resulting from its quadratic dependence on $j_{\alpha j}$ and the fact that $j_{\alpha j}$ contains three terms proportional respectively to $\nabla Y, \nabla T$ and $\nabla p$ (see equation (2.10)).

Illustrated in figure 8 are contour plots of the transitional-state $\log _{10}(\chi)$ for $\mathrm{HN} 600$, $\mathrm{HN} 800$, OH750 and OHe600; the HN800 case is added to the three simulations examined in tables 5-9 because the magnitude of the dissipation achieved at the HN800 transitional state matches the maximum obtained for the $\mathrm{OH}$ cases, namely for OH750 (figure 8 in Okong'o \& Bellan 2004a). Because either the perturbation wavelengths were different in the simulations (see table 2) which were thus performed in domains of different size, or because the mixing region represented a different portion of the domain, the presented figure size is not the same for all cases because in each case it maintains the aspect ratio of the significant portion of the flow. In all cases, the filamentary aspect of the atmospheric-presssure scalar dissipation is present. According to the r.m.s. of terms in the scalar dissipation analysed by Okong'o \& Bellan (2002a), the largest scalar gradient magnitude corresponds to regions of highest $\left(\nabla Y_{2} \cdot \nabla Y_{2}\right)$ value, and intermediate magnitudes correspond to high $\left(\nabla Y_{2} \cdot \nabla T\right)$ values, as the Fick/Soret cross-term was identified to be second in order of magnitude in $\chi$. Visually, the multitude of convoluted filaments for HN600 and OHe600 contrast with the smaller number of smooth filaments for HN800 and OH750 which is conjectured to result from the larger dissipation in the latter cases that reduces the gradient magnitude. In all cases, a thick prominent filament is observed in the upper stream corresponding to these HDGM regions mainly created from species mixing, and a thinner prominent filament is identifiable in the lower stream primarily corresponding to the distortion of the original density boundary (Okong'o \& Bellan $2004 a$ ). Generally, the interior of the layer contains filamentary structures of various thicknesses intermingled with thick patches of very small $\chi$. In fact, in the $\mathrm{OH} 750$ case, very small values of $\chi$ exist in the layer core, indicating minimal values of $\nabla Y_{2}$.

There are at least two ways for calculating the $\chi$ PDF: either by computing the PDF over the entire domain ( $\mathrm{Su} \&$ Clemens 2003) or by computing it over thin domain slices at fixed $x_{2}$ locations. The latter computation method was adopted here because the former was not conducive to understanding the flow features. The PDF of the scalar dissipation, $P(\chi)$, is illustrated in figure 9 as $P\left(\log _{10}(\chi)\right)$ computed over three-dimensional domain slices of thickness $\delta_{\omega, 0}$ centred at $x_{2} / \delta_{\omega, 0}=-5,0$ and 5 . Information is presented for HN600 (figures $9 a, 9 b$ and $9 c$ ), OH750 (figures $9 d, 9 e$ and $9 f$ ) and OHe600 (figures $9 g, 9 h$ and $9 i$ ) as representative of the entire database. At $x_{2} / \delta_{\omega, 0}=-5$ (figures $9 a, 9 d$ and $9 g$ ), all PDFs show a double peak with the peak at 
(a)
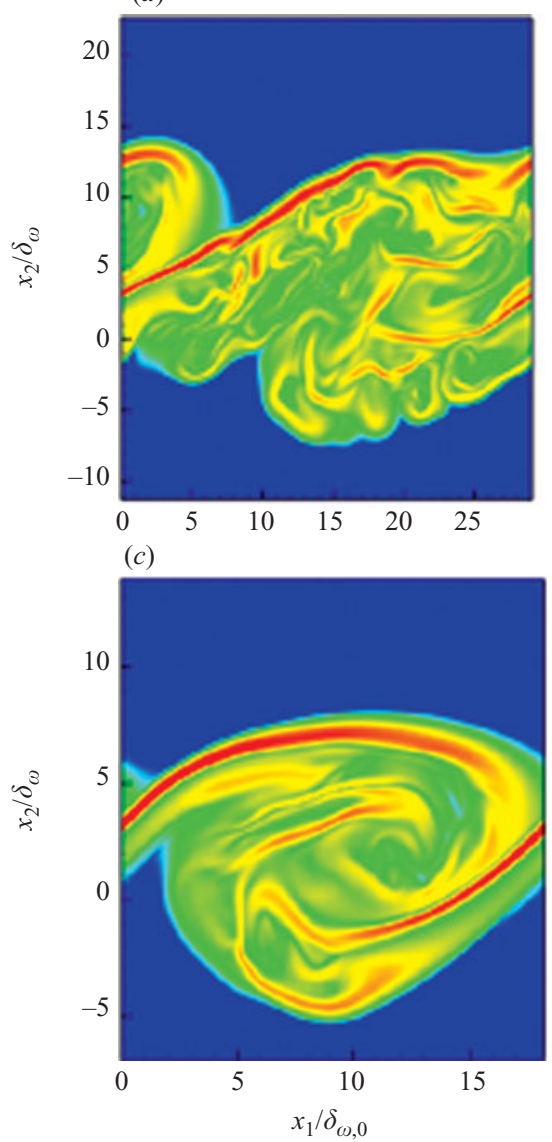

(b)
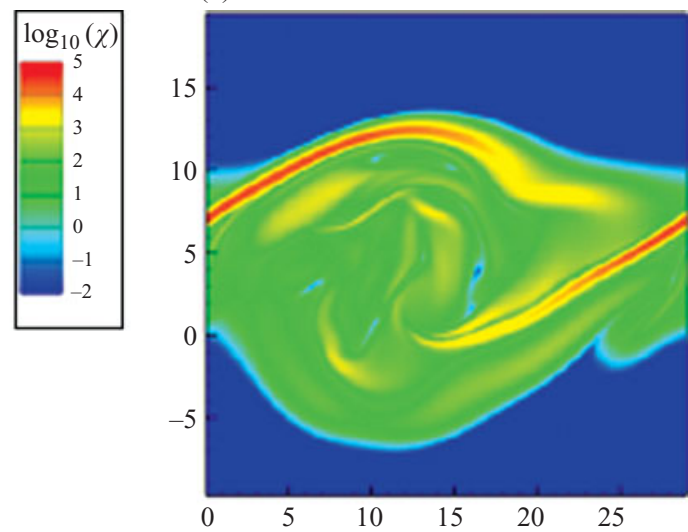

(d)

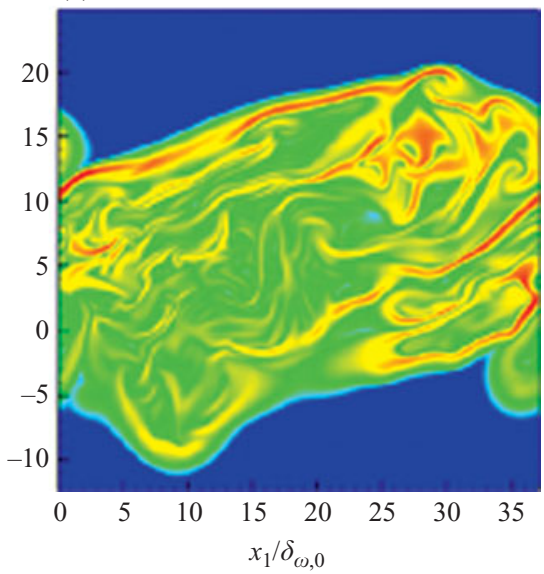

FIGURE 8. Scalar dissipation distribution in the $\left(x_{1}, x_{2}\right)$ between-the-braid plane $\left(x_{3} / L_{3}=0.5\right)$ at $t_{t r}^{*}$. (a) HN600, (b) OH750, (c) HN800 and $(d) \mathrm{OHe} 600$.

the smaller $\log _{10}(\chi)$ value attenuating from HN600 to OH750 and further to OHe600; the peak at the smaller $\log _{10}(\chi)$ value is indicative that the sampling picks up freestream regions of well-mixed fluid which seem to dominate for HN600 at $x_{2} / \delta_{\omega, 0}=-5$ (the peak at the smaller $\log _{10}(\chi)$ value is larger in magnitude). Unsurprisingly, the Gaussian is not a good approximation in this region of the flow. At $x_{2} / \delta_{\omega, 0}=0$ (figures $9 b, 9 e$ and $9 h$ ), a major peak exists, with minor local peaks past the mean scalar dissipation value. Except for OHe600 for which no minor peaks are detected, the Gaussian is not a reasonable approximation for the PDF; even for OHe600, the Gaussian suffers from the well-known inability to replicate the PDF at the largest values of $\log _{10}(\chi)$. Finally, at $x_{2} / \delta_{\omega, 0}=5$, the situation becomes exacerbated as far as the multitude of peaks is concerned, particularly at the largest $\log _{10}(\chi)$ values, for which local peaks are observed in all simulations, making again the Gaussian an inappropriate approximation. The existence of secondary peaks is entirely related to the scalar dissipation non-uniformity across the domain over which it was computed, particularly highlighting the HDGM regions that are the site of large gradients. Among the three examples in figure 9, the more prominent minor peaks are for OH750 for which the contours of the scalar dissipation displayed in figure 8 show 

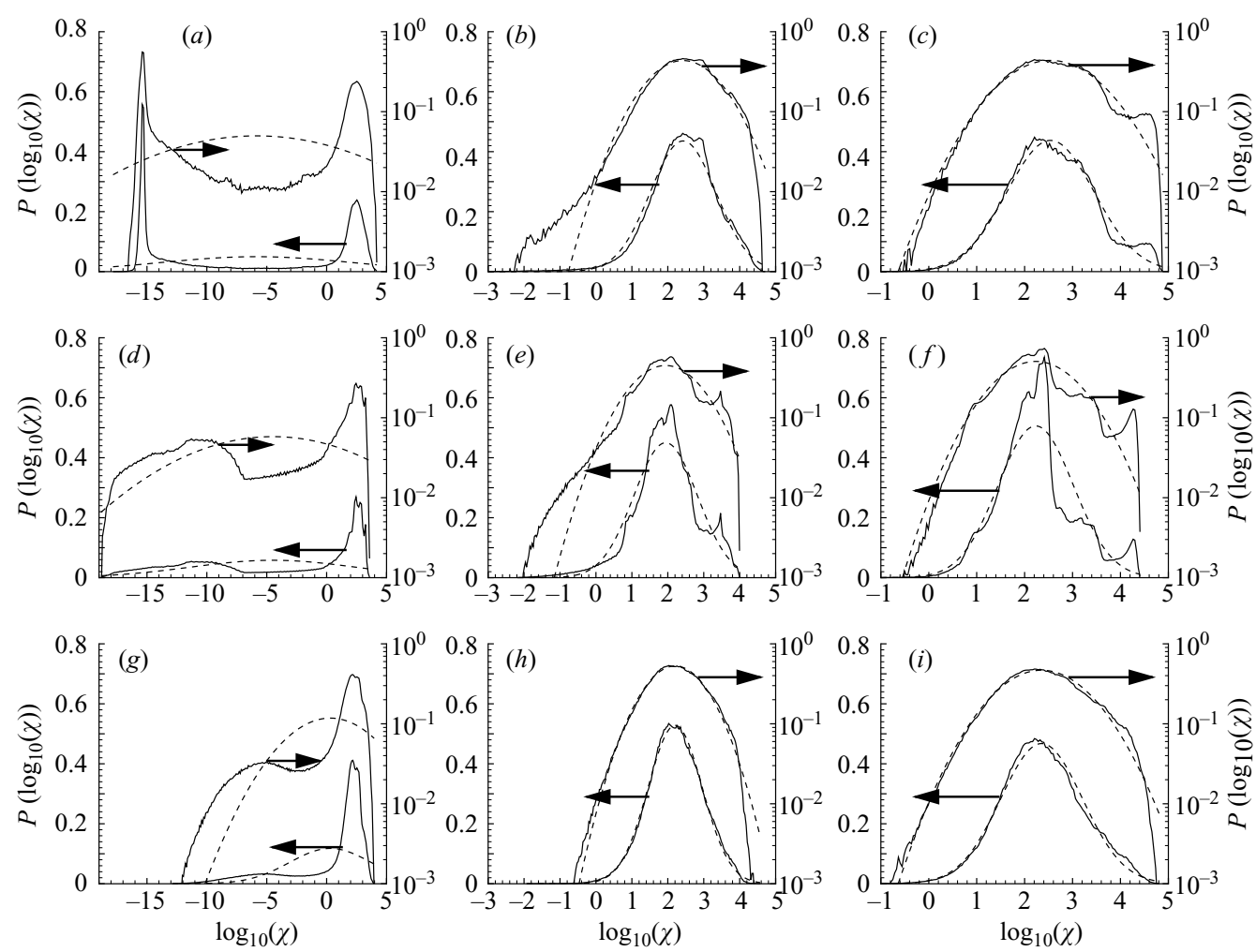

FIgURE 9. Scalar dissipation PDF at $t_{t r}^{*}$ for $\mathrm{HN} 600(a, b, c), \mathrm{OH} 750(d, e, f)$ and OHe600 $(g, h, i)$ at $x_{2} / \delta_{\omega, 0}=-5(a, d, g), x_{2} / \delta_{\omega, 0}=0(b, e, h), x_{2} / \delta_{\omega, 0}=5(c, f, i)$. In each case, the scalar dissipation PDF was calculated over one $\delta_{\omega, 0}$ unit centred at the specified value of $x_{2} / \delta_{\omega, 0} .-$, DNS extracted PDF; - - , Gaussian calculated with the mean and standard deviation of the DNS-extracted PDF. In each figure, the same data are plotted using two different ordinate axes. The arrows point to the ordinate axis relevant for the curve.

a central region of moderate magnitude with only two concentrated regions of large magnitude.

The far-from-Gaussian scalar dissipation provides an additional indication that the specific supercritical SGS models derived in $\S 4.1 .2$ and $\S 4.2$ rather than the traditional SGS models (e.g. no $(\overline{p(\phi)}-p(\bar{\phi}))$ or $\left(\overline{\boldsymbol{q}_{I K}(\phi)}-\boldsymbol{q}_{I K}(\bar{\phi})\right)$ models) will be necessary to duplicate the peculiar aspects of the $\chi$ PDF for the conditions of the DNS database. (To evaluate the validity of the Gaussian-based $\chi$ PDF flamelet models for reactive supercritical flows, experimental data are necessary to indicate if these flows also exhibit HDGM regions.)

\section{Summary and conclusions}

Large-eddy simulation (LES) models have been derived for fluids obeying a real-gas equation of state (EOS). Derived through filtering of the direct numerical simulation (DNS) equations, the LES equations contain unclosed terms that cannot be computed directly from the filtered flow field. Using an existing DNS database of supercritical binary-species temporal mixing layer simulations, explicit models for the SGS fluxes and simplifying assumptions for the remaining unclosed terms were assessed a priori. 
The DNS database consists of transitional states of high-pressure heptane/nitrogen, oxygen/hydrogen and oxygen/helium layers. The various assumptions were found to be generally valid, with two major exceptions: the gradient of the difference between the filtered pressure and the pressure computed on the filtered field was not negligible in the momentum equation, and the gradient of the difference between the filtered heat flux and the heat flux computed from the filtered field was a significant contribution in the energy equation. The importance of these two terms is a major departure from perfect-gas atmospheric gaseous flows where Okong'o \& Bellan (2004b) found them negligible. It was shown that there is a direct correspondence between these two new important terms and the high density-gradient-magnitude (HDGM) regions found both in DNS at transitional states and in experiments under fully turbulent conditions; the magnitude of the experimentally observed gradients is the same or exceeds that in DNS, showing that higher Reynolds number turbulence does not smear these gradients. Modelling was thus focused on these two novel terms as well as on the SGS fluxes.

To model the new terms, a Taylor expansion of the EOS was employed. The pressure-term model was shown to fulfil its function at a filter size that is linearly four times the DNS grid (the LES volume is 64 times that of the DNS); however, when the linear filter size is doubled (the LES volume is 512 times that of the DNS), the model is no longer acceptable, totally consistent with Taylor series principles. The implication is that numerical resolution will play an increasing role compared to that for atmospheric flows. Models derived for the heat flux were not entirely satisfactory a priori, and further a posteriori studies are necessary to determine whether such an approach is computationally efficient in a LES.

For modelling the SGS fluxes, constant-coefficient versions of Smagorinsky (SM), Gradient (GR) and Scale-Similarity (SS) models were assessed and calibrated on the DNS database. The SM model showed poor correlation with the exact SGS fluxes, while the GR and SS models had high correlations. Furthermore, the calibrated coefficients for the GR and SS models yielded good quantitative agreement with the SGS fluxes. However, comparison among the layers in the DNS database revealed that, statistically speaking, the calibrated coefficients were not generally valid. The more promising GR model was tested for computing the variances arising in the Taylor expansion of the pressure-term model, and it was found to have an excellent correlation with their DNS form. Calibrating coefficients were computed which were shown to be statistically significant at the two filter sizes for each simulation, but not across the entire database for a given filter size. However, the coefficients were statistically significant for simulations with a different set of binary species that reached similar momentum-thickness-based Reynolds number.

As a manifestation of small-scale mixing, the scalar dissipation was examined to investigate whether it displays departures from atmospheric-pressure flows that would require duplication in LES through the derived supercritical SGS models. The scalar dissipation distribution aspect was filamentary (like in atmospheric-pressure flows) and as expected, closely associated with the HDGM regions. Because the scalardissipation PDF computed in thin cross-stream layers did not generally exhibit the well-known quasi-Gaussian behaviour of atmospheric-pressure flows, the indications are that the specific supercritical SGS models will be essential in duplicating the peculiarities of small-scale mixing in supercritical flows.

Future studies will focus on a posteriori assessment of the LES models to determine their predictive ability in reproducing the temporal and spatial evolution of the filtered flow field, with particular interest in the sensitivity of the results to the value of the SGS-flux and SGS-variance model coefficients. 
This work was conducted at the Jet Propulsion Laboratory (JPL), California Institute of Technology (Caltech) and sponsored by the Air Force Office of Scientific Research under the direction of Dr Julian Tishkoff, by the Army Research Office under the direction of Dr David Mann, both through interagency agreements with the National Aeronautics and Space Administration (NASA), by the group SAFRAN that provided Caltech funds (to J. B.) for a Post Doctoral Fellow (L. C. S.), and by the NASA Fluid Microgravity Program (in a collaborative effort with the Air Force Research Laboratory at Edwards Air Force Base led by Dr Douglas Talley) under the direction of Dr Walter Duval. The authors are grateful to Professor Corin Segal from the University of Florida and to Dr Michael Oschwald from the German Aerospace Center (DLR) for graciously providing their data. The computational resources were provided by the JPL Supercomputing Center.

\section{Appendix A. Transport properties for $\mathrm{O}_{2} / \mathrm{He}$ mixtures}

For $\mathrm{O}_{2} / \mathrm{He}$ mixtures, the Prandtl number is approximated as

$$
\operatorname{Pr}=0.68+0.0283 \xi-0.5017 \xi^{2}-0.5390 \xi^{3}+\Delta P r,
$$

where

$$
\xi=\min \left(0.5, Y_{2}-0.81 \theta^{0.35}\right), \quad \theta=(T-100) / 800, \quad 0 \leqslant \theta \leqslant 1(T \text { in Kelvin }) .
$$

For $0.02 \leqslant \theta \leqslant 0.368, \Delta P r=2.42 Y_{2}^{14.6} \max (0.0,-0.23(1+\ln \theta))$, otherwise $\Delta P r=0$.

For $\mathrm{O}_{2} / \mathrm{He}$ mixtures, the Schmidt number is approximated as

$$
\begin{aligned}
S c & =\Sigma\left(Y_{2}\right)\left[1+(114 / T)^{1.5}\right] /\left(1+\Delta_{s}\right), \\
T & <200 K: \Sigma=\left(1.292-0.757 Y_{2}+0.444 Y_{2}^{2}-0.757 Y_{2}^{3}\right), \\
T & >200 K: \Sigma=\left(1.318-0.772 Y_{2}+0.453 Y_{2}^{2}-0.772 Y_{2}^{3}\right) .
\end{aligned}
$$

For $p<30 \mathrm{MPa}, \Delta_{s}=\min \left(0.08,0.1264+0.226 Y_{R}\right)+0.1 \exp \left(-2400 \theta^{4.5}\right)$ where $Y_{R}=Y_{2}-\min \left(1,0.5+0.78 \theta^{0.6}\right)$, otherwise $\Delta_{s}=0$.

\section{Appendix B. Derivatives of the PR EOS}

The relation between first-order derivatives with respect to the thermodynamic variables $\left(\rho, \rho Y_{1}, \rho Y_{2}, \rho e\right)$ and $\left(v, X_{1}, X_{2}, T\right)$ is

$$
\left(\begin{array}{c}
\left.\frac{\partial}{\partial \rho}\right|_{\rho Y_{i}, \rho e} \\
\left.\frac{\partial}{\partial\left(\rho Y_{1}\right)}\right|_{\rho, \rho Y_{2}, \rho e} \\
\left.\frac{\partial}{\partial\left(\rho Y_{2}\right)}\right|_{\rho, \rho Y_{1}, \rho e} \\
\left.\frac{\partial}{\partial(\rho e)}\right|_{\rho, \rho Y_{i}}
\end{array}\right)=\boldsymbol{M}\left(\begin{array}{c}
\left.\frac{\partial}{\partial v}\right|_{X_{i}, T} \\
\left.\frac{\partial}{\partial X_{1}}\right|_{v, X_{2}, T} \\
\left.\frac{\partial}{\partial X_{2}}\right|_{v, X_{1}, T} \\
\left.\frac{\partial}{\partial T}\right|_{v, X_{i}}
\end{array}\right) \text {, }
$$

which is succinctly written as

$$
\partial_{i}^{y}=\sum_{k=1}^{4} M_{i k} \partial_{k}^{x} .
$$



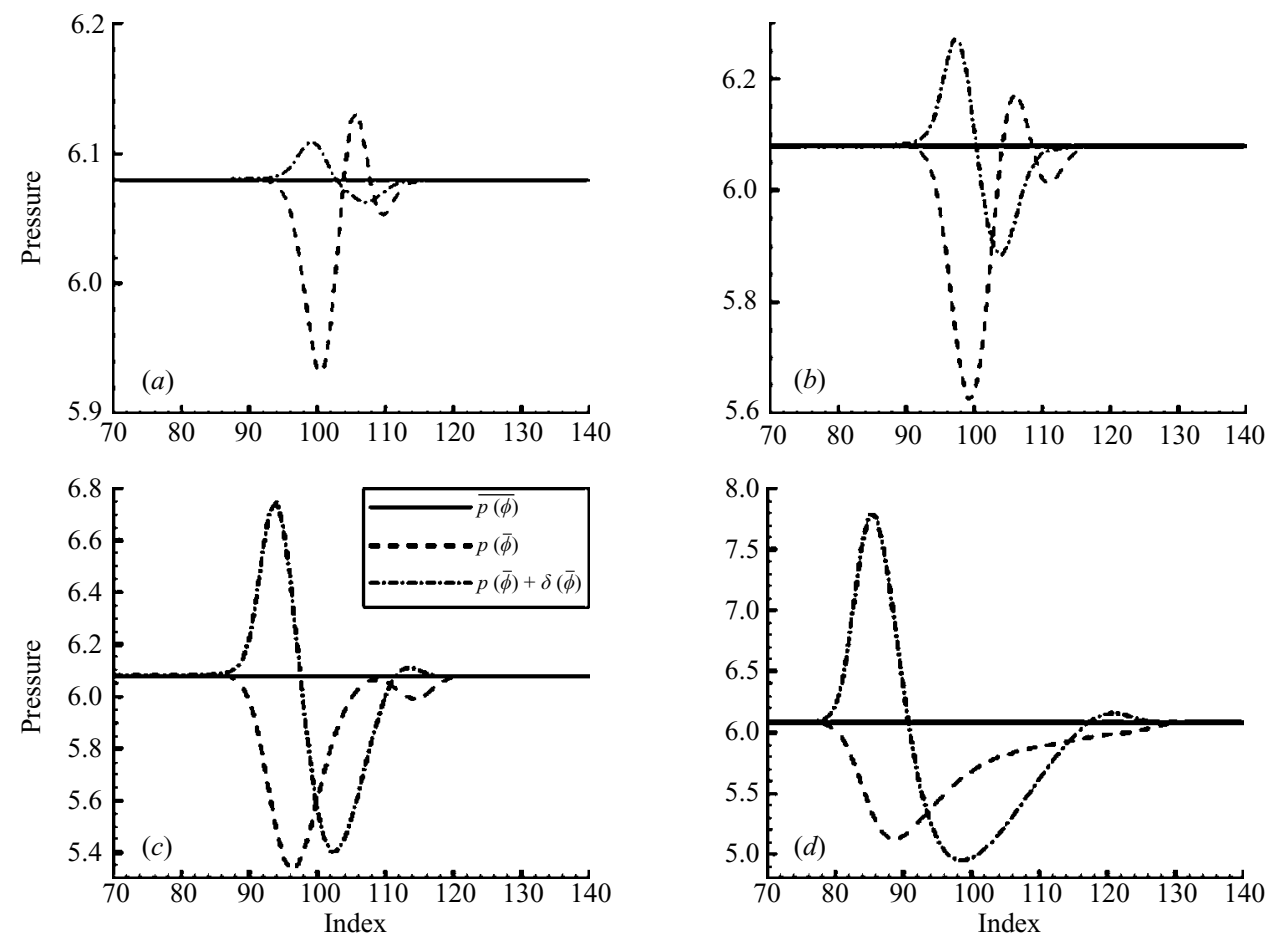

FIgURE 10. Plots from the one-dimensional PR EOS test. Improvement due to $\delta$ for (a) $r=1,(b) r=2,(c) r=3,(d) r=4$. Units are MPa.

Tedious calculations using the PR EOS lead to

$$
\boldsymbol{M}=v\left[\begin{array}{cccc}
0 & 0 & 0 & 0 \\
-\frac{v}{m_{1}} & \frac{1-X_{1}}{m_{1}} & -\frac{X_{2}}{m_{1}} & -\frac{\left[\partial(m h) / \partial X_{1}\right] \kappa_{T}-T \alpha_{v}\left[\partial v / \partial X_{1}\right]}{m_{1}\left(m C_{p}\right) \kappa_{S}} \\
-\frac{v}{m_{2}} & -\frac{X_{1}}{m_{2}} & \frac{1-X_{2}}{m_{2}} & -\frac{\left[\partial(m h) / \partial X_{2}\right] \kappa_{T}-T \alpha_{v}\left[\partial v / \partial X_{2}\right]}{m_{2}\left(m C_{p}\right) \kappa_{S}} \\
0 & 0 & 0 & \frac{\kappa_{T}}{\left(m C_{p}\right) \kappa_{S}}
\end{array}\right],
$$

where the isothermal compressibility, $\kappa_{T}$, the expansivity, $\alpha_{v}$, and the isentropic compressibility, $\kappa_{s}$, are

$$
\kappa_{T}=\frac{-1}{v(\partial p / \partial v)_{T, X}}, \quad \alpha_{v}=-\frac{(\partial p / \partial T)_{v, X}}{v(\partial p / \partial v)_{T, X}}, \quad \kappa_{s}=\kappa_{T}-v T \alpha_{v}^{2} /\left(m C_{p}\right) .
$$

In contracted form, the calculation of the second-order derivatives can be written as

$$
\partial_{i j}^{y}=\sum_{k=1}^{4} M_{i k} \partial_{k}^{x}\left[\sum_{l=1}^{4} M_{j l} \partial_{l}^{x}\right]=\sum_{k=1}^{4} \sum_{l=1}^{4} M_{i k}\left\{M_{j l} \partial_{k l}^{x}+\partial_{k}^{x}\left[M_{j l}\right] \partial_{l}^{x}\right\},
$$

which uses the derivatives with respect to $\left(v, X_{k}, T\right)$ of the matrix $\boldsymbol{M}$ (term $\left.\partial_{k}^{x}\left[M_{j l}\right]\right)$. These derivatives have lengthy mathematical expressions which are not provided here in the interest of brevity, but can be made available by the authors on request. 


\section{Appendix C. One-dimensional test for the Taylor expansion of $\overline{p(\phi)}$}

We investigate here in a simplified geometry whether $p(\bar{\phi})+\delta$ is a better approximation of $\overline{p(\phi)}$ than $p(\bar{\phi})$. The non-dimensional parameter assessing the $\delta$ improvement is the ratio $r=\bar{\Delta} / \Delta_{\text {grad }}$ of the filter size to the gradient scale. The test is performed on mathematical forms of $T$ and $X$ that mimic the initial condition in DNS, being a tanh profile between two (free-stream) specified values, while $p$ is a constant. Comparisons between $\overline{p(\phi)}, p(\bar{\phi})$ and $p(\bar{\phi})+\delta$ for 4 values of $r$ are shown in figure 10. For the small values of $r$ (figures $10 a$ and 10b) it is clear that $p(\bar{\phi})+\delta$ is a better approximation of $\overline{p(\phi)}$ than $p(\bar{\phi})$. Not only is the amplitude of the unphysical oscillations reduced, but more importantly their mean is much closer to zero. However, for the cases with a larger ratio $r$ (figures $10 c$ and $10 d$ ), the addition of $\delta$ actually deteriorates the correlation with $\overline{p(\phi)}$. These facts are consistent with the nature of the second-order correction provided by the Taylor expansion, valid for small variations (i.e. $(\phi-\bar{\phi}) \longrightarrow 0)$.

\section{REFERENCES}

Bardina, J., Ferziger, J. \& Reynolds, W. 1980 Improved subgrid scale models for large eddy simulation. AIAA Paper 80-1357.

Bellan, J. 2006 Theory, modeling and analysis of turbulent supercritical mixing. Combust. Sci. Technol. 178, 253-281.

Chehroudi, B., Talley, D. \& Coy, E. 1999 Initial growth rate and visual characteristics of a round jet into a sub- to supercritical environment of relevance to rocket, gas turbine and diesel engines. AIAA Paper 99-0206.

Clark, R., Ferziger, J. \& Reynolds, W. 1979 Evaluation of subgrid-scale models using an accurately simulated turbulent flow. J. Fluid Mech. 91, 1-16.

Gonzales-Bagnoli, M. G., Shapiro, A. A. \& Stenby, E. H. 2003 Evaluation of the thermodynamic models for the thermal diffusion factor. Phil. Mag. 83(17-18), 2171-2183.

Hannoun, I. A., Fernando, H. J. S. \& List, E. J. 1988 Turbulence structure near a sharp density interface. J. Fluid Mech. 189, 189-209.

Harstad, K., Miller, R. S. \& Bellan, J. 1997 Efficient high-pressure state equations. AIChE J. 43(6), 1605-1610.

Harstad, K. \& Bellan, J. 1998 Isolated fluid oxygen drop behavior in fluid hydrogen at rocket chamber pressures. Intl J. Heat Mass Transfer 41, 3537-3550.

Harstad, K. \& Bellan, J. 2000 An all-pressure fluid-drop model applied to a binary mixture: heptane in nitrogen. Intl J. Multiphase Flow 26(10), 1675-1706.

Harstad, K. \& Bellan, J. 2001 The $D^{2}$ variation for isolated LOX drops and polydisperse clusters in hydrogen at high temperature and pressures. Combust. Flame 124(4), 535-550.

Hirshfelder, J., Curtis, C. \& Bird, R. 1964 Molecular Theory of Gases and Liquids. John Wiley and Sons.

KeIzer, J. 1987 Statistical Thermodynamics of Nonequilibrium Processes. Springer.

Kennedy, C. \& CARPEnTER, M. 1994 Several new numerical methods for compressible shear layer simulations. Appl. Numer. Maths 14, 397-433.

Leboissetier, A., OKong'O, N. \& Bellan, J. 2005 Consistent large-eddy simulation of a temporal mixing layer laden with evaporating drops. Part 2. A Posteriori modelling. J. Fluid Mech. 523, 37-78.

LiU, S., Meneveau, C. \& Katz, J. 1994 On the properties of similarity subgrid-scale models as deduced from measurements in a turbulent jet. J. Fluid Mech. 275, 83-119.

Mayer, W., Schik, A., Schweitzer, C. \& Schaffler, M. 1996 Injection and mixing processes in high pressure LOX/GH2 rocket combustors. AIAA Paper 96-2620.

MaYer, W., Ivancic, B., Schik, A. \& Hornung, U. 1998 Propellant atomization in LOX/GH2 rocket combustors. AIAA Paper 98-3685.

Miller, R., Harstad, K. \& Bellan, J. 2001 Direct numerical simulations of supercritical fluid mixing layers applied to heptane-nitrogen. J. Fluid Mech. 436, 1-39. 
Moser, R. \& Rogers, M. 1991 Mixing transition and the cascade to small scales in a plane mixing layer. Phys. Fluids A 3(5), 1128-1134.

Moser, R. \& Rogers, M. 1993 The three-dimensional evolution of a plane mixing layer: pairing and transition to turbulence. J. Fluid Mech. 247, 275-320.

Muller, S. M. \& SCheERER, D. 1991 A method to parallelize tridiagonal solvers. Parallel Computing 17, 181-188.

Oefelein, J. C. \& Yang, V. 1998 Modeling high-pressure mixing and combustion processes in liquid rocket engines. J. Propul. Power 14, 843-857.

Oefelein, J. C. 2005 Thermophysical characteristics of shear-coaxial LOX-H2 flames at supercritical pressure. Proc. Combust. Inst. 30, 2929-2937.

OKong'o, N. \& Bellan, J. $2002 a$ Direct numerical simulation of a transitional supercritical binary mixing layer: heptane and nitrogen. J. Fluid Mech. 464, 1-34.

OKong'o, N. \& Bellan, J. 2002b Consistent boundary conditions for multicomponent real gas mixtures based on characteristic waves. J. Comput. Phys. 176, 330-344.

OKONG'O, N. \& BELLAN, J. 2003 Real gas effects of mean flow and temporal stability of binary-species mixing layers. AIAA J. 41(12), 2429-2443.

OKong'o, N. \& Bellan, J. $2004 a$ Turbulence and fluid-front area production in binary-species, supercritical, transitional mixing layers. Phys. Fluids 16(5), 1467-1492.

OKong'o, N. \& Bellan, J. 2004b Consistent large eddy simulation of a temporal mixing layer laden with evaporating drops. Part 1 . Direct numerical simulation, formulation and a priori analysis. J. Fluid Mech. 499, 1-47.

Okong'o, N., Harstad, K., \& Bellan, J. 2002 Direct numerical simulations of $\mathrm{O}_{2} / \mathrm{H}_{2}$ temporal mixing layers under supercritical conditions. AIAA J. 40(5), 914-926.

OschWald, M. \& SchiK, A. 1999 Supercritical nitrogen free jet investigated by spontaneous Raman scattering. Exps. Fluids 27, 497-506.

Oschwald, M., SchiK, A. KlaR, M. \& MaYer, W. 1999 Investigation of coaxial LN2/GH2-injection at supercritical pressure by spontaneous Raman scattering. AIAA Paper 99-2887.

Papamoschou, D. \& Roshko, A. 1988 The compressible turbulent shear layer: an experimental study. J. Fluid Mech. 197, 453-477.

Peters, N. 2000 Turbulent Combustion. Cambridge University Press.

Polikhov, S. \& Segal, C. 2007 Subcritical to supercritical jet mixing. AIAA Paper 2007-0569.

Prausnitz, J., Lichtenthaler, R. \& De Azevedo, E. 1986 Molecular Thermodynamics for FluidPhase Equilibrium. Prentice-Hall.

Pruett, C., Sochacki, J. \& Adams, N. 2001 On Taylor-series expansions of residual stress. Phys. Fluids 13(9), 2578-2589.

Selle, L. C. \& Bellan, J. 2007 Scalar dissipation modeling for passive and active scalars: a priori study using Direct Numerical Simulation. Proc. Comb. Inst. 31, 1665-1673.

SMAGORINKSY, J. 1993 Some historical remarks on the use of nonlinear viscosities. In Large Eddy Simulation of Complex Engineering and Geophysical Flows (ed. B. Galperin \& S. Orszag), chap. 1, pp. 3-36. Cambridge University Press.

Su, L. K. \& Clemens, N. T. 2003 The structure of fine-scale scalar mixing in gas-phase planar turbulent jets. J. Fluid Mech. 488, 1-29.

Vreman, B., Geurts, B. \& Kuerten, H. 1995 A priori tests of large eddy simulation of the compressible plane mixing layer. J. Engng Maths 29, 299-327.

Warhaft, Z. 2000 Passive scalars in turbulent flows. Annu. Rev. Fluid Mech. 32, 203-240.

Yoshizawa, A. 1986 Statistical theory for compressible turbulent shear flows, with the application to subgrid modeling. Phys. Fluids 29(7), 2152-2164.

Zong, N., Meng, H., Hsien, S.-Y. \& YANG, V. 2004 A numerical study of cryogenic fluid injection and mixing under supercritical conditions. Phys. Fluids 16(12), 4248-4261. 